\title{
STABILITY OF SASAKI-EXTREMAL METRICS UNDER COMPLEX DEFORMATIONS
}

\author{
CRAIG VAN COEVERING
}

\begin{abstract}
We consider the stability of Sasaki-extremal metrics under deformations of the transversal complex structure of the Sasaki foliation $\mathscr{F}_{\xi}$, induced by the Reeb vector field $\xi$. Let $g$ be a Sasaki-extremal metric on $M$, $G$ a compact connected subgroup of the automorphism group of the Sasaki structure, and suppose the reduced scalar curvature satisfies $s_{g}^{G}=0$. And consider a $G$-equivariant deformation $\left(\mathscr{F}_{\xi}, \bar{J}_{t}\right)_{t \in \mathcal{B}}$ of of the transversely holomorphic foliation preserving $\mathscr{F}_{\xi}$ as a smooth foliation. Provided the Futaki invariant relative to $G$ of $g$ is nondegenerate, the existence of Sasaki-extremal metrics is preserved under small variations of $t \in \mathcal{B}$ and of the Reeb vector $\xi \in \mathfrak{z}$ in the center of $\mathfrak{g}$. If $G=T \subseteq \operatorname{Aut}(g, \xi)$ is a maximal torus, the nondegeneracy of the Futaki invariant is automatic. So such deformations provide the easiest examples.

When the initial metric $g$ is Sasaki-Einstein this result can be improved using known properties of the Futaki invariant Although the relative Futaki invariant is useless in this case, non-trivial deformations can be obtained when $G=T \subseteq \operatorname{Aut}(g, \xi)$ is a maximal torus. Then a slice of the above family of Sasaki-extremal metrics is Sasaki-Einstein. Thus for each $t \in \mathcal{B}$ there is a $\xi_{t} \in \mathfrak{z}$ so that the Sasaki-extremal metric with Reeb vector field $\xi_{t}$ is Sasaki-Einstein. We apply this to deformations of toric 3-Sasaki 7-manifolds to obtain new families of Sasaki-Einstein metrics on these manifolds, which are deformations of 3-Sasaki metrics.
\end{abstract}

\section{INTRODUCTION}

Recall that a polarization on a complex manifold $M$ is and element $\Omega \in H^{1,1}(M) \cap$ $H^{2}(M, \mathbb{R})$ such that $\Omega$ can be represented by a Kähler form $\omega_{g}$ of a Kähler metric $g$ on $M$. In the hope of finding a canonical metric in the polarization E. Calabi [7, 8] defined a natural Riemannian functional on this space of Kähler metrics. Denote by $\mathfrak{M}_{\Omega}$ the space of Kähler metrics representing the polarization. Calabi proposed that one should seek critical points of the functional

$$
\begin{aligned}
\mathfrak{M}_{\Omega} & \stackrel{\mathfrak{C}}{\longrightarrow} \mathbb{R} \\
g & \mapsto \int_{M} s_{g}^{2} d \mu_{g}
\end{aligned}
$$

where $s_{g}$ is the scalar curvature of $g$ and $d \mu_{g}$ the volume form. He called the critical points of this functional extremal Kähler metrics and showed that $g$ is extremal if and only if the gradient of $s_{g}$ is a real holomorphic vector field. In particular, a constant scalar curvature metric is extremal, but many examples of extremal metrics are known which are not constant scalar curvature. An extremal Kähler metric is of constant scalar curvature if and only if the Futaki invariant

Date: April 5, 2012.

1991 Mathematics Subject Classification. Primary 53C25, Secondary 32Q20.

Key words and phrases. Sasaki metrics, extremal metrics, deformation, Sasaki-Einstein. 
vanishes [14, 8]. Many examples are known of extremal metrics of both constant and nonconstant scalar curvature

One way of producing new examples is to start with a known extremal metric and try to deform the solution as either the Kähler class or complex structure varies. This has been done with considerable success by C. LeBrun and S. R. Simanca [19, 20], where it was shown that there is no obstruction to deforming extremal metrics as the Kähler class is varied, whereas a nondegeneracy condition on the Futaki invariant is sufficient to guarantee that a constant scalar curvature metric can be deformed through extremal metrics as the complex structure is deformed. The nondegeneracy of the Futaki invariant is necessary as deforming the complex structure can result in a reduction of the size of the automorphism group. Later Y. Rollin, S. R. Simanca, and C. Tipler [25] generalized the later result to the case of equivariant deformations of the complex structure, where the sufficient condition becomes the nondegeneracy of the relative Futaki invariant.

This article gives analogous results for Sasaki manifolds. Similar results as in [25] are proved, although the a polarization of a Sasaki manifold is given by a choice of Reeb vector field, rather than a Kähler class. Thus the notions of the Sasaki polarization and nondegeneracy of the relative Futaki invariant involve varying the Reeb vector field.

1.1. Main result. Sasaki geometry sits between two Kähler geometries. If $(M, g)$ has is Sasaki then the metric cone $\left(C(M)=\mathbb{R}_{>0} \times M, \bar{g}=d r^{2}+r^{2} g\right)$ is Kähler for some almost complex structure. Furthermore, a Sasaki structure is contact and the foliation $\mathscr{F}_{\xi}$ generated by the Reeb vector field $\xi$ is transversely Kähler, i.e. locally the transversal space to the leaves has a complex structure $\bar{J}$ so that the induced metric $g^{T}$ is Kähler. Alternatively, $\bar{J}$ is an integrable almost complex structure on $\nu\left(\mathscr{F}_{\xi}\right)=T M / \tau\left(\mathscr{F}_{\xi}\right)$, where $\tau\left(\mathscr{F}_{\xi}\right) \subset T M$ is the subbundle tangent to the leaves.

So it is not surprising that the notion of extremal metric can be defined analogously for Sasaki metrics using the same functional (1) defined on the space $\mathfrak{M}(\xi, \bar{J})$ of metrics arising from Sasaki structures with Reeb vector field $\xi$ and transversal complex structure $\bar{J}$. This program was carried out in [5]. See also [6]. Not surprisingly, critical points are Sasaki metrics $g$ with the gradient of $s_{g}$ a transversally real holomorphic vector field. One notable difference from the Kähler case is the role of the polarization $\Omega$ is taken by the Reeb vector field $\xi$. The stability of extremal solutions under variations of $\xi$ was proved by C. P. Boyer, K. Galicki, and S. R. Simanaca [5].

The goal of this article is to give a similar stability result for Sasaki-extremal metrics under equivariant deformations of the transversal complex structure to the Reeb foliation. The results we obtain are similar to those in 25 in the Kähler case. Let $(g, \eta, \xi, \Phi)$ is a Sasaki-extremal structure on $M$. Then as in the Kähler case 8, it was shown in [5] that the identity component of automorphism group of the Sasaki structure $\operatorname{Aut}(g, \eta, \xi, \Phi)_{0}$ is a maximal compact subgroup of $\operatorname{Fol}\left(M, \mathscr{F}_{\xi}, \bar{J}\right)_{0}$, the identity component of the group of transversely holomorphic automorphisms of the foliation $\mathscr{F}_{\xi}$. Let $G \subseteq G^{\prime}=\operatorname{Aut}(g, \eta, \xi, \Phi)_{0}$ be a connected subgroup with Lie algebras $\mathfrak{g} \subseteq \mathfrak{g}^{\prime}$ so that $\xi \in \mathfrak{g}$. Then $\mathfrak{g}^{\prime} /\{\xi\} \subseteq \mathfrak{h}^{T}(\xi, \bar{J})_{0}$, where $\mathfrak{h}^{T}(\xi, \bar{J})_{0}$ is the subspace of transversely holomorphic vector fields modulo those tangent to the leaves, in $\tau\left(\mathscr{F}_{\xi}\right)$, that have holomorphy potentials, i.e. are of the form $\partial^{\#} \phi:=(\bar{\partial} \phi)^{\#}$ for a basic function $\phi$. Let $\mathfrak{z}=Z(\mathfrak{g})$ be the center of $\mathfrak{g}$ and $\mathfrak{z}^{\prime}=C_{\mathfrak{g}^{\prime}}(\mathfrak{g})$ the centralizer 
of $\mathfrak{g}$ in $\mathfrak{g}^{\prime}$. Also define $\mathfrak{p}=N_{\mathfrak{g}^{\prime}}(\mathfrak{g})$ to be the normalizer of $\mathfrak{g}$ in $\mathfrak{g}^{\prime}$. It will turn out that $\mathfrak{p} / \mathfrak{g}=\mathfrak{z}^{\prime} / \mathfrak{z}$.

Denote the space of $G$-invariant smooth functions by $C^{\infty}(M)^{G}$. A transversal deformation of the Sasaki structure $(g, \eta, \xi, \Phi)$ is a Sasaki structure $(\tilde{g}, \tilde{\eta}, \xi, \tilde{\Phi})$ with $\tilde{\eta}=\eta+d^{c} \phi$ for $\phi \in C^{\infty}(M)^{G}$ and transversal Kähler form $\tilde{\omega}^{T}=\omega^{T}+\frac{1}{2} d d^{c} \phi$, while the Reeb vector field and transversal complex structure $\bar{J}$ remain unchanged. We can then introduce the notion of the reduced scalar curvature $s_{g}^{G}$ for any $G$-invariant Sasaki structure, and the Futaki invariant relative to $G$

$$
\mathcal{F}_{G, \xi}: \mathfrak{p} / \mathfrak{g} \rightarrow \mathbb{R}
$$

where $\mathfrak{p}$ is the normalizer of $\mathfrak{g}$ in $\mathfrak{g}^{\prime}$, which is independent of the Sasaki structure with Reeb vector field $\xi$ and transversal complex structure $\bar{J}$. This space of Sasaki structure we denote $\mathcal{S}(\xi, \bar{J})$. On the space of $G$-invariant structures $\mathcal{S}(\xi, \bar{J})^{G}$ the condition $s_{g}^{G}=0$ is equivalent to $g$ being Sasaki-extremal and $\mathcal{F}_{G, \xi} \equiv 0$.

The connected component of the identity of the center is a torus $T^{r} \subseteq G$, and the contact structure defines a moment map on the cone $C(M)=\mathbb{R}_{>0} \times M$,

$$
\mu_{\eta}: C(M) \rightarrow \mathfrak{z}^{*},
$$

where $\mu_{\eta}(x, r)(X)=r^{2} \eta_{x}\left(X_{x}\right)$, with $X \in \mathfrak{z}$ and $X_{x}$ the induced vector at $x \in M$. The image of (2) is a convex polyhedral cone $\mathcal{C}_{\mathfrak{z}}^{*} \subset \mathfrak{z}^{*}([10])$. Although $\mu_{\eta}$ obvious depends on $\eta$, the image $\mathcal{C}_{\mathfrak{z}}^{*}$ is the same for any transversal deformation $\tilde{\eta}=\eta+d^{c} \phi$, for $\phi \in C^{\infty}(M)_{G}$, and turns out to be independent of a choice of Reeb vector fields in $\mathfrak{z}$. Define $\mathfrak{z}^{+}=\{\zeta \in \mathfrak{z}: \eta(\zeta)>0\}$. If $\zeta \in \mathfrak{z}^{+}$, then $\eta_{\zeta}=\eta(\zeta)^{-1} \eta$ is easily seen to be a contact form of a Sasaki structure with the same CR structure as $(\xi, \tilde{\eta}, \tilde{\Phi}, \tilde{g})$ and with Reeb vector field $\zeta$. Fakas' theorem says the dual cone $\mathcal{C}_{\mathfrak{z}}$ to $\mathcal{C}_{\mathfrak{z}}^{*}$ is a convex polyhedral cone, and from (2) we see that

$$
\mathfrak{z}^{+}=\stackrel{\circ}{\mathcal{C}}_{\mathfrak{z}} .
$$

Differentiating the relative Futaki invariant with $\xi$ varying in $\mathfrak{z}$ induces a linear map

$$
\mathfrak{p} / \mathfrak{g} \rightarrow \mathfrak{z}^{*},
$$

and we say that the Futaki invariant $\mathcal{F}_{G, \xi}$ relative to $G$ is nondegenerate if it is injective.

We consider $G$-equivariant deformations of the transversal complex structure of the Reeb foliation $\left(\mathscr{F}_{\xi}, \bar{J}\right)$. We fix the smooth structure of $\mathscr{F}_{\xi}$, so a deformation is given by $\left(\mathscr{F}_{\xi}, \bar{J}_{t}\right)_{t \in \mathcal{B}}$. The holomorphic structure on $\mathscr{F}_{\xi}$ has a versal deformation space 12, 17, with tangent space

$$
H_{\bar{\partial}_{b}}^{1}\left(\mathcal{A}^{0, \bullet}\right), \quad \text { where } \mathcal{A}^{0, k}=\Gamma\left(\Lambda_{b}^{0, k} \otimes \nu\left(\mathscr{F}_{\xi}\right)^{1,0}\right)
$$

denotes the basic $(0, k)$-forms with values in $\nu\left(\mathscr{F}_{\xi}\right)^{1,0}$ and

$$
0 \longrightarrow \mathcal{A}^{0,0} \stackrel{\bar{\partial}_{b}}{\longrightarrow} \mathcal{A}^{0,1} \stackrel{\bar{\partial}_{b}}{\longrightarrow} \cdots
$$

is the basic Dolbeault complex with values in the transverse holomorphic bundle $\nu\left(\mathscr{F}_{\xi}\right)^{1,0}$. Then $H_{\bar{\partial}_{b}}^{1}\left(\mathcal{A}^{0, \bullet}\right)^{G}$ is the tangent space to the $G$-equivariant deformations of $\left(\mathscr{F}_{\xi}, \bar{J}\right)$. By [1] the transversely Kähler property of $\left(\mathscr{F}_{\xi}, \bar{J}\right)$ is stable under small deformations. But the existence of a compatible Sasaki structure 
may be obstructed. The obstruction, due to H. Nozawa [22, is reviewed in Section 3.1. An unobstructed deformation $\left(\mathscr{F}_{\xi}, \bar{J}_{t}\right)_{t \in \mathcal{B}}$ is said to be of $(1,1)$-type. If $\mathcal{B}$ is smooth, after possibly restricting to a neighborhood of zero in $\mathcal{B}$, there is a family $\left(g_{t}, \eta_{t}, \xi, \Phi_{t}\right) \in \mathcal{S}\left(\xi, \bar{J}_{t}\right), t \in \mathcal{B}$. And if $\left(\mathscr{F}_{\xi}, \bar{J}_{t}\right)_{t \in \mathcal{B}}$ is $G$-equivariant, we may assume that the family $\left(g_{t}, \eta_{t}, \xi, \Phi_{t}\right), t \in \mathcal{B}$ is $G$-equivariant. In particular, if $\operatorname{Ric}_{g}>0$ then the obstruction vanishes on a neighborhood of zero in any deformation.

Associated to the family $\left(\mathscr{F}_{\xi}, \bar{J}_{t}\right)_{t \in \mathcal{B}}$ for sufficiently small $\phi \in C^{\infty}(M)^{G}$ we consider the Sasaki metrics $g_{t, \alpha, \phi}$ with transverse Kähler form

$$
\omega_{t, \alpha, \phi}^{T}=\omega_{t, \alpha}^{T}+\frac{1}{2} d d^{c} \phi
$$

with Reeb vector field $\xi+\alpha \in \mathfrak{z}^{+}, \eta_{t, \alpha, \phi}=\eta_{t, \xi+\alpha}+d^{c} \phi$ and $\omega_{t, \alpha}^{T}=\frac{1}{2} d \eta_{t, \xi+\alpha}$. Thus we have a family of Sasaki metrics parametrized by a neighborhood of $(0,0,0) \in$ $\mathcal{B} \times \mathfrak{z} \times C^{\infty}(M)^{G}$. Assuming that $g_{0,0,0}$ is Sasaki-extremal and satisfies $s_{g_{0,0}, 0}^{G}=0$, we seek solutions to $s_{g_{t, \alpha, \phi}}^{G}=0$ for $(t, \alpha, \phi)$ close to zero. Using suitable Banach spaces, an application of the implicit function theorem gives the main theorem.

Theorem 1. Let $(g, \xi, \eta, \Phi)$ be a Sasaki-extremal structure satisfying $s_{g}^{G}=0$. Suppose $G \subseteq G^{\prime}=\operatorname{Aut}(\xi, \eta, \Phi, g)_{0}$ is a connected compact subgroup and $\left(\mathscr{F}_{\xi}, \bar{J}_{t}\right)_{t \in \mathcal{B}}$ a $G$-equivariant deformation of $(1,1)$-type. If the Futaki invariant relative to $G$ is nondegenerate $g$, then there is a small neighborhood of zero $W \subset \mathcal{B} \times \mathfrak{z} \times C^{\infty}(M)^{G}$ and a smooth closed submanifold $V \subset W$, with $\operatorname{dim} V=\operatorname{dim}_{\mathbb{R}} \mathcal{B}+\operatorname{dim} \mathfrak{z}$ so that for $(t, \alpha, \phi) \in V$ there is Sasaki metric $g_{t, \alpha, \phi}$ satisfying $s_{g_{t, \alpha, \phi}}^{G}=0$. Therefore, there is a space of Sasaki-extremal metrics parametrized by $V$. Furthermore, the projection $\pi: V \rightarrow \mathcal{B}$ is a submersion with fibers of dimension $\operatorname{dim} \mathfrak{z}$.

Unfortunately, the nondegeneracy of the relative Futaki invariant is not an easy condition to work with, and from (3) we see that $\mathfrak{z}$ must be sufficiently large in $\mathfrak{g}$ for it to hold. Fortunately, if $G=T \subset G^{\prime}$ is a maximal torus, then the relative Futaki invariant is trivially nondegenerate as $\mathfrak{p} / \mathfrak{g}=0$.

Corollary 2. Let $(g, \eta, \xi, \Phi)$ be a Sasaki-extremal structure satisfying $s_{g}^{G}=0$. Suppose that $\left(\mathscr{F}_{\xi}, \bar{J}_{t}\right)_{t \in \mathcal{B}}$ is a $G$-equivariant deformation of $(1,1)$-type, where $G \subseteq$ $G^{\prime}=\operatorname{Aut}(g, \eta, \xi, \Phi)_{0}$ is a maximal torus. Then there is a neighborhood of zero $W \subset \mathfrak{B} \times \mathfrak{g}$, so that for $(t, \alpha) \in W$ there is Sasaki metric $g_{t, \alpha, \phi_{t, \alpha}}$ satisfying $s_{g_{t, \alpha, \phi_{t, \alpha}}^{G}}^{G}=0$. So for each fixed $t \in \mathcal{B}$ close to zero, the space of extremal metrics is locally parametrized by a neighborhood of zero in $\mathfrak{g}$.

Just as in the Kähler-Einstein case [19] the linear map (3) is always trivial when $(g, \eta, \xi, \Phi)$ is Sasaki-Einstein. Fortunately, Corollary 2 is still useful in obtaining new examples of Sasaki-Einstein metrics when $G$ is a maximal torus. In this case one can use the nondegeneracy of the Futaki invariant on $\mathfrak{g}$ [21, 16] to show that there is a neighborhood $W \subset \mathcal{B} \times \mathfrak{g}$ so that for each $(t, 0) \in W$ there is a $\alpha_{t} \in \mathfrak{g}$ so that $g_{t, \alpha_{t}, \phi_{t}}$ is Sasaki-Einstein.

Corollary 3. Let $(g, \eta, \xi, \Phi)$ be a Sasaki-Einstein structure, and suppose that $\left(\mathscr{F}_{\xi}, \bar{J}_{t}\right)_{t \in \mathcal{B}}$ is a $G$-equivariant deformation, where $G \subseteq G^{\prime}=\operatorname{Aut}(g, \eta, \xi, \Phi)_{0}$ is a maximal torus. Then there is a neighborhood $U \subset \mathcal{B}$ so that for $t \in U$ there is a unique $\alpha_{t} \in \mathfrak{g}$ and $a \phi_{t} \in C^{\infty}(M)^{G}$ so that $g_{t, \alpha_{t}, \phi_{t}}$ is Sasaki-Einstein. 
In the final section Corollary 3 is applied to give a new family of Sasaki-Einstein metrics by deforming the underlying Sasaki-Einstein metric on toric 3-Sasaki 7manifolds. These manifolds were studied in [4] as torus 3-Sasaki quotients of spheres where it was proved that there are infinitely many of each Betti number $b_{2}(M) \geq 1$. Note that they are not toric as Sasaki-Einstein manifolds. If $b_{2}(M) \geq 2$, then for a fixed Sasaki structure $G=T^{3}=\operatorname{Aut}(g, \xi, \eta, \Phi)_{0}$ is a 3-torus. It was calculated by the author in 29] that $H_{\bar{\partial}_{b}}^{1}\left(\mathcal{A}^{0, \bullet}\right)=H_{\bar{\partial}_{b}}^{1}\left(\mathcal{A}^{0, \bullet}\right)^{T^{3}}=b_{2}(M)-1$ giving a smooth versal deformation space. Thus Corollary2 2 gives a neighborhood $W \subset \mathbb{C}^{b_{2}(M)-1} \times \mathfrak{g}$ of zero parametrizing a space of Sasaki-extremal metrics. And furthermore, Corollary 3 gives a slice of Sasaki-Einstein metrics. There is a neighborhood $U \subset \mathbb{C}^{b_{2}(M)-1}$ so that for $t \in U$ there is an $\alpha_{t} \in \mathfrak{g}$ and $\phi_{t} \in C^{\infty}(M)^{G}$ so that $g_{t, \alpha_{t}, \phi_{t}}$ is SasakiEinstein and contained in a real 3-dimensional space of Sasaki-extremal metrics. Note that the Einstein metrics in this family have three different isometry groups as shown in Figure 1.

These examples provide the first examples, to the author's knowledge, of deformations of 3-Sasaki metrics to metrics which are Sasaki-Einstein but not 3-Sasaki. These are also examples of Einstein manifolds admitting 3 Killing spinors with deformations to Einstein metrics admitting only 2 Killing spinors. More details will appear in 29.

1.2. Acknowledgements. I would like to thank the Max Planck Institute for Mathematics for their hospitality and excellent research environment that I enjoyed while writing this article.

\section{BACKGROUND}

\subsection{Sasaki manifolds.}

Definition 4. A Riemannian manifold $(M, g)$ is a Sasaki manifold, or has a compatible Sasaki structure, if the metric cone $(C(M), \bar{g})=\left(\mathbb{R}_{>0} \times M, d r^{2}+r^{2} g\right)$ is Kähler with respect to some complex structure $I$, where $r$ is the usual coordinate on $\mathbb{R}_{>0}$.

Thus $M$ is odd and denoted $n=2 m+1$, while $C(M)$ is a complex manifold with $\operatorname{dim}_{\mathbb{C}} C(M)=m+1$.

Although, this is the simplest definition, Sasaki manifolds were originally defined as a special type of metric contact structure. See the monograph [3] or [16] for more details on the properties of Sasaki manifolds that we summarize below. We will identify $M$ with the $\{1\} \times M \subset C(M)$. Let $r \partial_{r}$ be the Euler vector field on $C(M)$, then it is easy to see that $\xi=I r \partial_{r}$ is tangent to $M$. Using the warped product formulae for the cone metric $\bar{g} 24$ it is easy check that $r \partial_{r}$ is real holomorphic, $\xi$ is Killing with respect to both $g$ and $\bar{g}$, and furthermore the orbits of $\xi$ are geodesics on $(M, g)$. Define $\left.\eta=\frac{1}{r^{2}} \xi\right\lrcorner \bar{g}$, then we have

$$
\eta=-\frac{I^{*} d r}{r}=d^{c} \log r
$$

where $d^{c}=\sqrt{-1}(\bar{\partial}-\partial)$. If $\omega$ is the Kähler form of $\bar{g}$, i.e. $\omega(X, Y)=\bar{g}(I X, Y)$, then $\mathcal{L}_{r \partial_{r}} \omega=2 \omega$ which implies that

$$
\left.\omega=\frac{1}{2} d\left(r \partial_{r}\right\lrcorner \omega\right)=\frac{1}{2} d\left(r^{2} \eta\right)=\frac{1}{4} d d^{c}\left(r^{2}\right) .
$$


From (5) we have

$$
\omega=r d r \wedge \eta+\frac{1}{2} r^{2} d \eta
$$

We will use the same notation to denote $\eta$ and $\xi$ restricted to $M$. Then (6) implies that $\eta$ is a contact form with Reeb vector field $\xi$, since $\eta(\xi)=1$ and $\mathcal{L}_{\xi} \eta=0$. Let $D \subset T M$ be the contact distribution which is defined by

$$
D_{x}=\operatorname{ker} \eta_{x}
$$

for $x \in M$. Furthermore, if we restrict the almost complex structure to $D, J:=\left.I\right|_{D}$, then $(D, J)$ is a strictly pseudoconvex CR structure on $M$. We have a splitting of the tangent bundle $T M$

$$
T M=D \oplus L_{\xi},
$$

where $L_{\xi}$ is the trivial subbundle generated by $\xi$. It will be convenient to define a tensor $\Phi \in \operatorname{End}(T M)$ by $\left.\Phi\right|_{D}=J$ and $\Phi(\xi)=0$. Then

$$
\Phi^{2}=-\mathbb{1}+\eta \otimes \xi \text {. }
$$

Since $\xi$ is Killing, we have

$$
d \eta(X, Y)=2 g(\Phi(X), Y), \quad \text { where } X, Y \in \Gamma(T M),
$$

and $\Phi(X)=\nabla_{X} \xi$, where $\nabla$ is the Levi-Civita connection of $g$. Making use of (9) we see that

$$
g(\Phi X, \Phi Y)=g(X, Y)-\eta(X) \eta(Y),
$$

and one can express the metric by

$$
g(X, Y)=\frac{1}{2}(d \eta)(X, \Phi Y)+\eta(X) \eta(Y) .
$$

We will denote a Sasaki structure on $M$ by $(g, \eta, \xi, \Phi)$. Although, the reader can check that merely specifying $(g, \xi),(g, \eta)$, or $(\eta, \Phi)$ is enough to determine the Sasaki structure, it will be convenient to denote the remaining structure.

The Reeb foliation $\mathscr{F}_{\xi}$ on $M$ generated by the action of $\xi$ will be important in the sequel. Note that it has geodesic leaves and is a Riemannian foliation, that is has a $\xi$ invariant Riemannian metric on the normal bundle $\nu\left(\mathscr{F}_{\xi}\right)$. But in general the leaves are not compact. If the leaves are compact, or equivalently $\xi$ generates an $S^{1}$-action, then $(g, \eta, \xi, \Phi)$ is said to be a quasi-regular Sasaki structure, otherwise it is irregular. If this $S^{1}$ action is free, then $(g, \eta, \xi, \Phi)$ is said to be regular. In this last case $M$ is an $S^{1}$-bundle over a manifold $Z$, which we will see below is Kähler. If the structure if merely quasi-regular, then the leaf space has the structure of a Kähler orbifold $Z$. In general, in the irregular case, the leaf space is not even Hausdorff but we will make use of the transversally Kähler property of the foliation $\mathscr{F} \xi$ which we discuss next.

2.2. Transverse Kähler structure. We now describe a transverse Kähler structure on $\mathscr{F}_{\xi}$. The vector field $\xi-\sqrt{-1} I \xi=\xi+\sqrt{-1} r \partial_{r}$ is holomorphic on $C(M)$. If we denote by $\tilde{\mathbb{C}}^{*}$ the universal cover of $\mathbb{C}^{*}$, then $\xi+\sqrt{-1} r \partial_{r}$ induces a holomorphic action of $\tilde{\mathbb{C}}^{*}$ on $C(M)$. The orbits of $\tilde{\mathbb{C}}^{*}$ intersect $M \subset C(M)$ in the orbits of the Reeb foliation generated by $\xi$. We denote the Reeb foliation by $\mathscr{F}_{\xi}$. This gives $\mathscr{F}_{\xi}$ a transversely holomorphic structure. 
The foliation $\mathscr{F}_{\xi}$ together with its transverse holomorphic structure is given by an open covering $\left\{U_{\alpha}\right\}_{\alpha \in A}$ and submersions $\pi_{\alpha}: U_{\alpha} \rightarrow W_{\alpha} \subset \mathbb{C}^{m}$ such that when $U_{\alpha} \cap U_{\beta} \neq \emptyset$ the map

$$
\phi_{\beta \alpha}=\pi_{\beta} \circ \pi_{\alpha}^{-1}: \pi_{\alpha}\left(U_{\alpha} \cap U_{\beta}\right) \rightarrow \pi_{\beta}\left(U_{\alpha} \cap U_{\beta}\right)
$$

is a biholomorphism.

Not that on $U_{\alpha}$ the differential $d \pi_{\alpha}: D_{x} \rightarrow T_{\pi_{\alpha}(x)} W_{\alpha}$ at $x \in U_{\alpha}$ is an isomorphism taking the almost complex structure $J_{x}$ to that on $T_{\pi_{\alpha}(x)} W_{\alpha}$. Since $\xi\lrcorner d \eta=0$ the 2-form $\frac{1}{2} d \eta$ descends to a form $\omega_{\alpha}^{T}$ on $W_{\alpha}$. Similarly, $g^{T}=\frac{1}{2} d \eta(\cdot, \Phi \cdot)$ satisfies $\mathcal{L}_{\xi} g^{T}=0$ and vanishes on vectors tangent to the leaves, so it descends to an Hermitian metric $g_{\alpha}^{T}$ on $W_{\alpha}$ with Kähler form $\omega_{\alpha}^{T}$. The Kähler metrics $\left\{g_{\alpha}^{T}\right\}$ and Kähler forms $\left\{\omega_{\alpha}^{T}\right\}$ on $\left\{W_{\alpha}\right\}$ by construction are isomorphic on the overlaps

$$
\phi_{\beta \alpha}: \pi_{\alpha}\left(U_{\alpha} \cap U_{\beta}\right) \rightarrow \pi_{\beta}\left(U_{\alpha} \cap U_{\beta}\right) .
$$

We will use $g^{T}$, respectively $\omega^{T}$, to denote both the Kähler metric, respectively Kähler form, on the the local charts and the globally defined pull-back on $M$.

If we define $\nu\left(\mathscr{F}_{\xi}\right)=T M / L_{\xi}$ to be the normal bundle to the leaves, then we can generalize the above concept. A tensor $\Psi \in \Gamma\left(\left(\nu\left(\mathscr{F}_{\xi}\right)^{*}\right)^{\otimes p} \otimes \nu\left(\mathscr{F}_{\xi}\right)^{\otimes q}\right)$ is basic if $\mathcal{L}_{V} \Psi=0$ for any vector field $V \in \Gamma\left(L_{\xi}\right)$. It is sufficient to check this for $V=\xi$. Then $g^{T}$ and $\omega^{T}$ are such tensors on $\nu\left(\mathscr{F}_{\xi}\right)$. We will also make use of the bundle isomorphism $\pi: D \rightarrow \nu\left(\mathscr{F}_{\xi}\right)$, which induces an almost complex structure $\bar{J}$ on $\nu\left(\mathscr{F}_{\xi}\right)$ so that $(D, J) \cong\left(\nu\left(\mathscr{F}_{\xi}\right), \bar{J}\right)$ as complex vector bundles. Clearly, $\bar{J}$ is basic and is mapped to the almost complex structure by local charts $d \pi_{\alpha}: D_{x} \rightarrow$ $T_{\pi_{\alpha}(x)} W_{\alpha}$.

To work on the Kähler leaf space we define the Levi-Civita connection of $g^{T}$ by

$$
\nabla_{X}^{T} Y= \begin{cases}\pi_{\xi}\left(\nabla_{X} Y\right) & \text { if } X, Y \text { are smooth sections of } D \\ \pi_{\xi}([V, Y]) & \text { if } X=V \text { is a smooth section of } L_{\xi},\end{cases}
$$

where $\pi_{\xi}: T M \rightarrow D$ is the orthogonal projection onto $D$. Then $\nabla^{T}$ is the unique torsion free connection on $D \cong \nu\left(\mathscr{F}_{\xi}\right)$ so that $\nabla^{T} g^{T}=0$. Then for $X, Y \in \Gamma(T M)$ and $Z \in \Gamma(D)$ we have the curvature of the transverse Kähler structure

$$
R^{T}(X, Y) Z=\nabla_{X}^{T} \nabla_{Y}^{T} Z-\nabla_{Y}^{T} \nabla_{X}^{T} Z-\nabla_{[X, Y]}^{T} Z
$$

and similarly we have the transverse Ricci curvature $\operatorname{Ric}^{T}$ and scalar curvature $s^{T}$. We will denote the transverse Ricci form by $\rho^{T}$.

The following follows from O'Neill tensor computations for a Riemannian submersion. See [23] and [1, Ch. 9].

Proposition 5. Let $(M, g, \eta, \xi, \Phi)$ be a Sasaki manifold of dimension $n=2 m+1$, then

(i) $\operatorname{Ric}_{g}(X, \xi)=2 m \eta(X), \quad$ for $X \in \Gamma(T M)$,

(ii) $\operatorname{Ric}^{T}(X, Y)=\operatorname{Ric}_{g}(X, Y)+2 g^{T}(X, Y)$, for $X, Y \in \Gamma(D)$,

(iii) $s^{T}=s_{g}+2 m$.

Definition 6. A Sasaki-Einstein manifold $(M, g, \eta, \xi, \Phi)$ is a Sasaki manifold with

$$
\operatorname{Ric}_{g}=2 m g \text {. }
$$


Note that by (ii) the Einstein constant must be $2 m$, and the transverse Kähler metric is also Einstein

$$
\operatorname{Ric}^{T}=(2 m+2) g^{T} .
$$

Conversely, if one has a Sasaki structure $(g, \eta, \xi, \Phi)$ with $\operatorname{Ric}^{T}=\tau g^{T}$ with $\tau>$ 0 , then after a $D$-homothetic transformation one has a Sasaki-Einstein structure $\left(g^{\prime}, \eta^{\prime}, \xi^{\prime}, \Phi\right)$, where $\eta^{\prime}=a \eta, \xi^{\prime}=a^{-1} \xi$, and $g^{\prime}=a g+a(a-1) \eta \otimes \eta$, with $a=\frac{\tau}{2 m+2}$.

Let $\mathcal{S}(\xi)$ be the space of Sasaki structures $(\tilde{g}, \tilde{\eta}, \tilde{\xi}, \tilde{\Phi})$ on $M$ with $\tilde{\xi}=\xi$. For any $(\tilde{g}, \tilde{\eta}, \tilde{\xi}, \tilde{\Phi}) \in \mathcal{S}(\xi)$ the 1 -form $\beta=\tilde{\eta}-\eta$ is basic, so $[d \tilde{\eta}]_{b}=[d \eta]_{b}$, where $[\cdot]_{b}$ denotes the basic cohomology class of a basic closed form. Thus $\left[\omega^{T}\right]_{b} \in H_{b}^{2}\left(M / \mathscr{F}_{\xi}, \mathbb{R}\right)$ is the same for every Sasaki structure in $\mathcal{S}(\xi)$. Thus, as first observed in [5], fixing the Reeb vector field is the closest analogue to a polarization in Kähler geometry, and we say that the Reeb vector field $\xi$ polarizes the Sasaki manifold.

For a fixed Reeb vector field $\xi$, we consider a fixed transversal complex structure on $\mathscr{F}_{\xi}$ which is equivalent to fixing $\bar{J}$ on $\nu(\mathscr{F} \xi)$. We define $\mathcal{S}(\xi, \bar{J}) \subset \mathcal{S}(\xi)$ to be the subset of Sasaki structures inducing the same complex normal bundle $(\nu(\mathscr{F} \xi), \bar{J})$, in other words, the set of $(\tilde{g}, \tilde{\eta}, \xi, \tilde{\Phi}) \in \mathcal{S}(\xi)$ such that the following diagram commutes

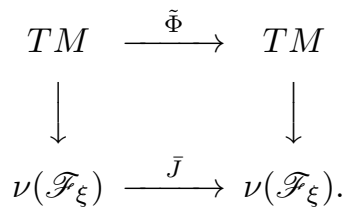

We will consider three different deformations of a Sasaki structure. First we consider transverse Kähler deformations.

Lemma 7 ([3, [5] $)$. The space $\mathcal{S}(\xi, \bar{J})$ of all Sasaki structures with Reeb vector field $\xi$ and transverse holomorphic structure $\bar{J}$ is an affine space modeled on $C_{b}^{\infty}(M) / \mathbb{R} \times$ $C_{b}^{\infty}(M) / \mathbb{R} \times H^{1}(M, \mathbb{R})$. If $(g, \eta, \xi, \Phi) \in \mathcal{S}(\xi, \bar{J})$ is a fixed Sasaki structure then another structure $(\tilde{g}, \tilde{\eta}, \tilde{\xi}, \tilde{\Phi}) \in \mathcal{S}(\xi, \bar{J})$ is determined by real basic functions $\phi$ and $\psi$ and an harmonic, with respect to $g$, 1-form $\alpha$ such that

$$
\begin{aligned}
\tilde{\eta} & =\eta+d^{c} \phi+d \psi+\alpha, \\
\tilde{\Phi} & =\Phi-\xi \otimes \tilde{\eta} \circ \Phi, \\
\tilde{g} & =\frac{1}{2} d \tilde{\eta} \circ(\mathbb{1} \otimes \tilde{\Phi})+\tilde{\eta} \otimes \tilde{\eta},
\end{aligned}
$$

and the transversal Kähler form becomes $\tilde{\omega}^{T}=\omega^{T}+\frac{1}{2} d d^{c} \phi$.

Proof. We give only a sketch. See 3 for details. The 1 -form $\gamma=\tilde{\eta}-\eta$ is basic, and since $d \gamma \in \Gamma\left(\Lambda_{b}^{1,1}\right)$ and $\gamma$ is real, $d^{c} d \gamma=0$. And we have the Hodge decomposition

$$
\gamma=d^{c} \phi+d \psi+\alpha,
$$

with respect to the transversal Kähler metric $g^{T}$, where $\alpha \in \mathcal{H}_{g^{T}}^{1}$ is harmonic. But note that $\mathcal{H}_{\mathbb{R}, g^{T}}^{1}=\mathcal{H}_{\mathbb{R}, g}^{1}$, where the latter is the space of real harmonic 1-forms on $(M, g)$. This is because a $\beta \in \Gamma\left(\Lambda^{1}(M)\right)$ satisfying $d \beta=0$ and $\mathcal{L}_{\xi} \beta=0$ must be basic.

Remark 2.8. It is easy to check that the parameter $\psi$ in (16) changes the structure only by a gauge transformation along the leaves. That is, if $\psi \in C_{b}^{\infty}(M)$, then $\exp (\psi \xi)^{*} \eta=\eta+d \psi$. 
2.3. transversely extremal metrics. Given a basic $\phi \in C_{b}^{\infty}(M, \mathbb{C})$, we define $\partial_{g}^{\#} \phi$ to be the $(1,0)$ component of the gradient, that is

$$
g\left(\partial_{g}^{\#} \phi, \cdot\right)=\bar{\partial} \phi .
$$

In order for $\partial_{g}^{\#} \phi$ to be transversely holomorphic we need in addition $\bar{\partial} \partial_{g}^{\#} \phi=0$. This is equivalent to the fourth-order transversally elliptic equation

$$
L_{g}^{b} \phi:=\left(\bar{\partial} \partial_{g}^{\#}\right)^{*} \bar{\partial} \partial_{g}^{\#} \phi
$$

As in the Kähler case we have

$$
\left.L_{g}^{b} \phi=\frac{1}{4}\left(\Delta_{b}^{2}+\left(\rho^{T}, d d^{c} \phi\right)+2\left(\partial s^{T}\right)\right\lrcorner \partial_{g}^{\#} \phi\right) .
$$

We define the space of holomorphy potentials to be $\mathcal{H}_{g}^{b}:=\operatorname{ker} L_{g}^{b}$.

We denote by $\mathfrak{M}(\xi, \bar{J})$ the metrics associated with Sasaki structures in $\mathcal{S}(\xi, \bar{J})$. We define the Calabi functional just as in (1) by

$$
\begin{aligned}
& \mathfrak{M}(\xi, \bar{J}) \stackrel{\mathcal{C}}{\longrightarrow} \mathbb{R} \\
& g \mapsto \int_{M} s_{g}^{2} d \mu_{g}
\end{aligned}
$$

We seek critical points of $\mathcal{C}$. Because $\mathcal{C}$ only depends on the deformation of the transversal Kähler metric $\tilde{\omega}^{T}=\omega+\frac{1}{2} d d^{c} \phi$ and not the other parameters in Lemma 7 and Proposition 5 , iii these critical points are the transversely extremal metrics. The Euler-Lagrange equation for $\mathcal{C}$ was worked out in 5 .

Proposition 9 ([5]). The fist derivative of $\mathcal{C}$ at $g$ along the path $\omega_{t}^{T}=\omega^{T}+t \frac{1}{2} d d^{c} \phi$ is

$$
\left.\frac{d}{d t} \mathcal{C}\left(g_{t}\right)\right|_{t=0}=-4 \int_{M} s_{g}\left(L_{g}^{b} \phi\right) d \mu_{g} .
$$

Definition 10. A Sasaki metric $g \in \mathfrak{M}(\xi, \bar{J})$ is extremal if it is a critical point of (21). Equivalently, the basic vector field $\partial_{g}^{\#} s_{g}$ is transversely holomorphic.

2.4. automorphism groups. We consider the relevant automorphism groups and Lie algebras associated to a Sasaki structure $(g, \eta, \xi, \Phi)$.

Consider first the strictly pseudo convex CR structure $(D, J)$. We denote the group of $\mathrm{CR}$ automorphisms by $\mathrm{CR}(D, J)$ and its Lie algebra by $\operatorname{cr}(D, J)$. A fundamental result of [26] classifies strictly pseudoconvex CR manifolds for which $\mathrm{CR}(D, J)$ acts nonproperly. We only need the result for compact $M$.

Theorem 11 ([26). Let $(M, D, J)$ be a compact strictly pseudoconvex $C R$ manifold. If $\mathrm{CR}(D, J)$ is not compact, then $(M, D, J)$ is $C R$ diffeomorphic to $\mathbb{S}^{2 m+1}$ with the standard $C R$ structure, in which case $\mathrm{CR}(D, J)=\mathrm{PSU}(m+1,1)$.

It is useful to have the following alternative characterization of Sasaki structures.

Proposition 12. Let $(M, D, J)$ be a strictly pseudoconvex manifold. If $\xi \in \mathfrak{c r}(D, J)$ is everywhere transversal to $D$, then, after possibly changing sign to $-\xi$, there is a unique Sasaki structure $(g, \eta, \xi, \Phi)$ with Reeb vector field $\xi$.

Proof. Let $\eta$ be the unique 1-form with $\operatorname{ker} \eta=D$ and $\eta(\xi)=1$. After possibly changing signs on $\xi$ and $\eta$ we have $\left.d \eta\right|_{D}>0$. Since $\xi$ preserves the distribution $D$, $\mathcal{L}_{\xi} \eta=0$ and $\xi$ is the Reeb vector field of $\eta$. Then one can define $\Phi$ by $\left.\Phi\right|_{D}=J$ and $\Phi(\xi)=0$, and one has $\mathcal{L}_{\xi} \Phi=0$. This latter condition and the integrability of $(D, J)$ implies that $(g, \eta, \xi, \Phi)$ with $g$ defined in (11) is Sasaki. See [3] for details. 
We have the subgroup of the diffeomorphism group preserving the foliation $\mathscr{F}_{\xi}$

$$
\operatorname{Fol}\left(M, \mathscr{F}_{\xi}\right)=\left\{\phi \in \operatorname{Diff}(M): \phi_{*} \mathscr{F}_{\xi} \subset \mathscr{F}_{\xi}\right\},
$$

with Lie algebra

$$
\mathfrak{f o l}\left(M, \mathscr{F}_{\xi}\right)=\left\{X \in \mathscr{X}(M):[X, \xi] \subset \Gamma\left(L_{\xi}\right)\right\} .
$$

Note that $\operatorname{Fol}\left(M, \mathscr{F}_{\xi}\right)$ is infinite dimensional as every $X \in \Gamma\left(L_{\xi}\right)$ is in $\mathfrak{f o l}\left(M, \mathscr{F}_{\xi}\right)$. Any $\phi \in \operatorname{Fol}\left(M, \mathscr{F}_{\xi}\right)$ induces a map of bundles $\phi_{*}: \nu\left(\mathscr{F}_{\xi}\right) \rightarrow \nu\left(\mathscr{F}_{\xi}\right)$. The subgroup of transversely holomorphic automorphism of $\mathscr{F}_{\xi}$ can be characterized as those which induce an automorphism of the complex bundle $(\nu(\mathscr{F} \xi), \bar{J})$

$$
\operatorname{Fol}\left(M, \mathscr{F}_{\xi}, \bar{J}\right):=\left\{\phi \in \operatorname{Fol}\left(M, \mathscr{F}_{\xi}\right): \phi_{*} \circ \bar{J}=\bar{J} \circ \phi_{*}\right\} .
$$

Note that this group is also infinite dimensional, since any section in $L_{\xi}$ has a 1 parameter group in $\operatorname{Fol}\left(M, \mathscr{F}_{\xi}, \bar{J}\right)$. We will denote the projection of $X \in \mathscr{X}(M)$ to a section of $\nu\left(\mathscr{F}_{\xi}\right)$ by $\bar{X} \in \Gamma\left(\nu\left(\mathscr{F}_{\xi}\right)\right)$.

2.4.1. Transversely holomorphic vector fields. The Lie algebra fol $(M, \mathscr{F} \xi, \bar{J})$ of $\operatorname{Fol}\left(M, \mathscr{F}_{\xi}, \bar{J}\right)$ will be called the space of transversely holomorphic vector fields. A transversely holomorphic vector field can be characterized more succinctly.

Proposition 13. Let $(g, \eta, \xi, \Phi)$ be any Sasaki structure with Reeb vector field $\xi$ and transversely holomorphic structure $\bar{J}$. Thus $\Phi$ satisfies (15). Then $X \in$ $\mathfrak{f o r}\left(M, \mathscr{F}_{\xi}, \bar{J}\right)$ if and only if

$$
\overline{[X, \Phi Y]}=\overline{J[X, Y]}
$$

for all $Y \in \mathscr{X}(M)$.

Note that (22) implies that $X \in \mathfrak{f o r}\left(M, \mathscr{F}_{\xi}\right)$; that is, it is automatically foliate. Also, condition (22) is equivalent to the $(1,0)$ vector field

$$
\Xi=\frac{1}{2}(\bar{X}-\sqrt{-1} \bar{J} \bar{X}) \in \Gamma\left(\nu\left(\mathscr{F}_{\xi}\right)\right)
$$

satisfying the transverse Cauchy-Riemann equations.

Since $\mathfrak{f o r}\left(M, \mathscr{F}_{\xi}, \bar{J}\right)$ is infinite dimensional we define $\mathfrak{h o l}^{T}(\xi, \bar{J})$ to be the image of

$$
\begin{aligned}
\mathfrak{f o r}\left(M, \mathscr{F}_{\xi}, \bar{J}\right) & \stackrel{\pi}{\longrightarrow} \Gamma\left(\nu\left(\mathscr{F}_{\xi}\right)\right) \\
X & \mapsto \bar{X}
\end{aligned}
$$

which is a finite dimensional complex Lie algebra. We will use $\mathfrak{h o l}^{T}(\xi, \bar{J})$ to denote both transversally holomorphic $(1,0)$ vector fields as in (23), or transversally real holomorphic vector fields depending on the context.

The subspace $\mathfrak{h o l}^{T}(\xi, \bar{J})_{0} \subseteq \mathfrak{h o l}^{T}(\xi, \bar{J})$ of sections with a zero will turn out to be a Lie subalgebra. As remarked in the proof of Lemma 7

$$
H_{b}^{1,0}\left(M / \mathscr{F}_{\xi}\right) \oplus H_{b}^{0,1}\left(M / \mathscr{F}_{\xi}\right)=\mathcal{H}_{g^{T}}^{1}=\mathcal{H}_{g}^{1},
$$

where on the left we have the basic Dolbeault cohomology, and we see that $H_{1}(M, \mathbb{Z}) \subset$ $\left(H_{b}^{1,0}\right)^{*}$ is a lattice. As in Kähler geometry, we have the Albanese variety

$$
\operatorname{Alb}(M, \xi, \bar{J})=\left(H_{b}^{1,0}\right)^{*} / H_{1}(M, \mathbb{Z})=H_{b}^{0}\left(M, \Omega_{b}^{1}\right)^{*} / H_{1}(M, \mathbb{Z}),
$$

and associated map

$$
\mu: M \rightarrow \operatorname{Alb}(M, \xi, \bar{J})
$$


which is a transversely holomorphic map if one considers $\operatorname{Alb}(M, \xi, \bar{J})$ to have the trivial foliation with the points as leaves. Explicitly, if $p_{0} \in M$ is a fixed point and $\beta_{1}, \ldots \beta_{k} \in H_{b}^{0}\left(M, \Omega_{b}^{1}\right)$ are a basis then

$$
\mu(p)=\left(\int_{p_{0}}^{p} \beta_{1}, \ldots, \int_{p_{0}}^{p} \beta_{k}\right)
$$

for any path $\gamma$ from $p_{0}$ to $p$.

Arguing just as in [19, Thm. 1] we see that the image of

$$
\partial_{g}^{\#}: \mathcal{H}_{g}^{b} \rightarrow \mathfrak{h o l}^{T}(\xi, \bar{J})
$$

is precisely $\mathfrak{h o l}^{T}(\xi, \bar{J})_{0}$. Furthermore, (25) induces a group homomorphism $\mu$ : $\operatorname{Fol}\left(M, \mathscr{F}_{\xi}, \bar{J}\right) \rightarrow \operatorname{Aut}(A l b(M, \xi, \bar{J}))$, with $\mathfrak{h o l}^{T}(\xi, \bar{J})_{0} \subseteq \mathfrak{h o l}^{T}(\xi, \bar{J})$ the ideal given by the kernel.

Similar to the Kähler case we have the following.

Lemma 14. If $X \in \mathfrak{h o l}^{T}(\xi, \bar{J})_{0}$, then $X=\partial_{g}^{\#} f$ for an imaginary function $f \in$ $\sqrt{-1} C_{b}^{\infty}(M)$ if and only if $\operatorname{Re} X$ is Killing for $g^{T}$. If this is so, then $V=\operatorname{Re} X \in$ $\Gamma\left(\nu\left(\mathscr{F}_{\xi}\right)\right)$ lifts to a vector field $\tilde{V} \in \mathfrak{a u t}(g, \eta, \xi, \Phi)$. Conversely, if $\tilde{V} \in \mathfrak{a u t}(g, \eta, \xi, \Phi)$, then $V=\pi(\tilde{V})=\operatorname{Re} \partial_{g}^{\#} f$, for the imaginary valued function $f=\sqrt{-1} \eta(\tilde{V})$.

Proof. Suppose $V=\operatorname{Re} \partial_{g}^{\#} f$ with $f$ imaginary valued. Then $\left.\frac{\sqrt{-1}}{2} \bar{J}^{*} d f=V\right\lrcorner g^{T}$, so $\left.\frac{\sqrt{-1}}{2} d f=V\right\lrcorner \omega^{T}$, which implies that $\mathcal{L}_{V} \omega^{T}=0$ and $V$ is Killing.

Suppose $V$ is Killing and $\partial_{g}^{\#} f=V-\sqrt{-1} \bar{J} V$, where $f=u+\sqrt{-1} v$. Then $\left.\frac{1}{2}\left(d u-\bar{J}^{*} d v\right)=V\right\lrcorner g^{T}$, which implies $\left.\frac{1}{2}\left(-\bar{J}^{*} d u-d v\right)=V\right\lrcorner \omega^{T}$. Since $\mathcal{L}_{V} \omega^{T}=0$, we have $d d^{c} u=0$ and $u$ must be constant.

If $V=\operatorname{Re} \partial_{g}^{\#} f$ with $f$ imaginary valued, choose $\tilde{V} \in \mathfrak{X}(M)$ with $\pi(\tilde{V})=V$ and $\eta(\tilde{V})=-\sqrt{-1} f$. Then

$$
\left.\left.\mathcal{L}_{\tilde{V}} \eta=d(\eta(\tilde{V}))+\tilde{V}\right\lrcorner d \eta=-\sqrt{-1} d f+\tilde{V}\right\lrcorner d \eta=0 .
$$

2.4.2. Real holomorphy potentials. It will be useful to have a description of real holomorphic transversal vector fields and potentials. Given a real $X \in \mathfrak{h o l}^{T}(\xi, \bar{J})$, let $\beta=X^{b}$. If $\nabla^{-} \beta$ denotes the $\bar{J}$-anti-invariant component of $\nabla^{T} \beta$. Then a basic $X \in \Gamma\left(\nu\left(\mathscr{F}_{\xi}\right)\right)$ is transversally holomorphic if and only if $\nabla^{-} X=0$. It follows that $d d^{c} \beta=0$, and we have the Hodge decomposition

$$
\beta=\beta_{h}+d u_{X}+d^{c} v_{X},
$$

where $\beta_{h}$ is harmonic, $u_{X}$ and $v_{X}$ are real functions, and $d^{c} v_{X}$ is coclosed. We have $X \in \mathfrak{h o l}^{T}(\xi, \bar{J})_{0}$, when $\beta_{h}=0$ in which case $f_{X}=u_{X}+\sqrt{-1} v_{X}$ is the holomorphy potential of $X^{1,0}$, i.e. $X^{1,0}=\partial_{g}^{\#} f_{X}$. Note that $X$ is Killing for $g^{T}$ if and only if $u_{X}$ is constant.

We define the real operator $\mathbb{\llbracket}_{g}^{b}$ by

$$
\mathbb{L}_{g}^{b} f=\left(\nabla^{-} d\right)^{*}\left(\nabla^{-} d\right) f .
$$

Then a real basic function $f$ satisfies $\mathbb{L}_{g}^{b} f=0$ if and only if $\operatorname{grad} f$ is real holomorphic. And every $X \in \mathfrak{h o l}^{T}(\xi, \bar{J})_{0}$ Killing with respect to $g^{T}$ can be written $X=\bar{J} \operatorname{grad} f$ for such an $f$. As shown in [25] we have

$$
\mathbb{L}_{g}^{b} f=\frac{1}{2} \Delta_{b}^{2} f+\frac{1}{2}\left(\rho^{T}, d d^{c} f\right)+\frac{1}{2}\left(d f, d s_{g}\right),
$$


and comparing with (20) is related to $L_{g}^{b}$ by

$$
2 L_{g}^{b} f=\mathbb{L}_{g}^{b} f+\frac{\sqrt{-1}}{2} \mathcal{L}_{\bar{J} \operatorname{grad} s_{g}} f .
$$

Lemma 15 ([25]). The space of real basic solutions of $\mathbb{L}_{g}^{b}$ coincides with the space of real basic solutions of $L_{g}^{b}$.

As in Lemma 14 we have the correspondence

$$
\begin{aligned}
\mathcal{H}_{g}^{b} \cap C_{b}^{\infty}(M, \mathbb{R}) & \stackrel{\sim}{\mathfrak{a u t}}(g, \eta, \xi, \Phi) \\
v & \longmapsto X \text { s.t. } \eta(X)=v, \text { and } \pi(X)=\frac{1}{2} \bar{J} \operatorname{grad} v .
\end{aligned}
$$

2.4.3. Automorphisms of Sasaki-extremal manifolds. We recall the structure of $\mathfrak{h o l}^{T}(\xi, \bar{J})$ when $(g, \eta, \xi, \Phi)$ is Sasaki-extremal [5] as it will important to what follows. The result is similar to the theorem of Calabi [8] on the automorphism group of a Kähler manifold with an extremal metric.

Theorem 16. Let $(g, \eta, \xi, \Phi) \in \mathcal{S}(\xi, \bar{J})$ be a Sasaki-extremal structure. Then we have the semidirect sum decomposition

$$
\mathfrak{h o l}^{T}(\xi, \bar{J})=\mathfrak{a} \oplus \mathfrak{h o l}^{T}(\xi, \bar{J})_{0},
$$

where $\mathfrak{a}$ is the Lie algebra of parallel, with respect to $g^{T}$, sections of $\nu\left(\mathscr{F}_{\xi}\right)$. And we also have

$$
\mathfrak{h o l}^{T}(\xi, \bar{J})_{0}=\mathfrak{k} \oplus \bar{J} \mathfrak{k} \oplus\left(\bigoplus_{\lambda>0} \mathfrak{h}^{\lambda}\right),
$$

where $\mathfrak{k}=\mathfrak{a u t}(g, \eta, \xi, \Phi) / \xi$ is the image under $\partial_{g}^{\#}$ of the imaginary valued functions in $\mathcal{H}_{g}^{b}$ and $\mathfrak{h}^{\lambda}=\left\{\bar{X} \in \mathfrak{h} \mathfrak{l}^{T}(\xi, \bar{J})_{0}:\left[\partial_{g}^{\#} s_{g}, \bar{X}\right]=\lambda \bar{X}\right\}$ and $\mathfrak{k} \oplus \bar{J} \mathfrak{k}=C_{\mathfrak{h o l}^{T}(\xi, \bar{J})_{0}}\left(\partial_{g}^{\#} s_{g}\right)$, the centralizer of $\partial_{g}^{\#} s_{g}$.

Furthermore, the connected component of the identity $G=\operatorname{Aut}(g, \eta, \xi, \Phi)_{0} \subset$ $\operatorname{Fol}\left(M, \mathscr{F}_{\xi}, \bar{J}\right)$ is a maximal compact connected subgroup. And any other maximal compact connected subgroup is conjugate to $G$ in $\operatorname{Fol}\left(M, \mathscr{F}_{\xi}, \bar{J}\right)$.

Proof. Everything but the final statement is proved in [5]. The last statement was proved in [8] in the Kähler case, and easily follows from the theory of finite dimensional Lie groups. It is not as simple in this case as $\operatorname{Fol}\left(M, \mathscr{F}_{\xi}, \bar{J}\right)$ is infinite dimensional, and furthermore is not even known to have a Fréchet Lie group structure.

Let $G^{\prime} \subset \operatorname{Fol}\left(M, \mathscr{F}_{\xi}, \bar{J}\right)$ be any maximal connected compact subgroup with Lie algebra $\mathfrak{g}^{\prime}$. By applying the familiar averaging argument using a Haar measure on $G^{\prime}$ to $(g, \eta, \xi, \Phi)$ we get a Sasaki structure $(\tilde{g}, \tilde{\eta}, \tilde{\xi}, \tilde{\Phi})$ with $G^{\prime}=\operatorname{Aut}(\tilde{g}, \tilde{\eta}, \tilde{\xi}, \tilde{\Phi})_{0}$.

It is proved in [22] that there exist an $f \in \operatorname{Fol}(M, \mathscr{F} \xi, \bar{J})$ so that $f^{*} \tilde{\eta}(\xi)=c \in$ $\mathbb{R}_{>0}$. This follows from a leaf wise version of Moser's argument which can be used to prove $\left.f^{*} \tilde{\eta}\right|_{L_{\xi}}=\left.c \eta\right|_{L_{\xi}}$. But since the averaging preserves the volume

$$
\begin{aligned}
\operatorname{Vol}(M, \tilde{g}) & =\frac{1}{m !} \int_{M} f^{*} \tilde{\eta} \wedge\left(\frac{1}{2} f^{*} d \tilde{\eta}\right)^{m} \\
& =\frac{1}{m !} \int_{M} c^{m+1} \eta \wedge\left(\frac{1}{2} d \eta\right)^{m} \\
& =c^{m+1} \operatorname{Vol}(M, g),
\end{aligned}
$$


so $c=1$. The second equality follows because $f^{*} \tilde{\eta}-c \eta$ is a basic form and an application of Stokes theorem. Therefore $f$ applied to $(\tilde{g}, \tilde{\eta}, \tilde{\xi}, \tilde{\Phi})$ gives a Sasaki structure $\left(g^{\prime}, \eta^{\prime}, \xi, \Phi^{\prime}\right) \in \mathcal{S}(\xi, \bar{J})$ with $\operatorname{Aut}\left(g^{\prime}, \eta^{\prime}, \xi, \Phi^{\prime}\right)_{0}$ conjugate to $G^{\prime}$ in $\operatorname{Fol}\left(M, \mathscr{F}_{\xi}, \bar{J}\right)$.

We have the continuous group homomorphism

$$
\Upsilon: \operatorname{Fol}\left(M, \mathscr{F}_{\xi}, \bar{J}\right) \rightarrow \operatorname{Aut}\left(\mathfrak{h o r}^{T}(\xi, \bar{J})_{0}\right),
$$

with $\Upsilon(\phi) \bar{X}=\phi_{*} \bar{X}$, where $\operatorname{Fol}\left(M, \mathscr{F}_{\xi}, \bar{J}\right)$ is given the topology as a closed subgroup of the diffeomorphism group. By considering 1-parameter subgroups generated by $X$ such that $\bar{X} \in \mathfrak{h o l}^{T}(\xi, \bar{J})_{0}$, one sees that the adjoint group of Lie algebra $\mathfrak{h o l}^{T}(\xi, \bar{J})_{0}, \quad H=\operatorname{Inn}\left(\mathfrak{h o l}^{T}(\xi, \bar{J})_{0}\right) \subseteq \operatorname{Aut}\left(\mathfrak{h o l}{ }^{T}(\xi, \bar{J})_{0}\right)$, is in the image of $\Upsilon$. Note that the Lie algebra $\mathfrak{h}$ of $H=\operatorname{Inn}\left(\mathfrak{h o r} r^{T}(\xi, \bar{J})_{0}\right)$ is $\mathfrak{h o l}{ }^{T}(\xi, \bar{J})_{0} / Z\left(\mathfrak{h o l} \mathfrak{l}^{T}(\xi, \bar{J})_{0}\right)$.

Let $G=\operatorname{Aut}(g, \eta, \xi, \Phi)_{0}$ for a Sasaki-extremal structure, then by (32) it is easy to see $G$ is maximal connected compact. Let $G^{\prime} \subset \operatorname{Fol}\left(M, \mathscr{F}_{\xi}, \bar{J}\right)$ be any other connected maximal compact subgroup with Lie algebra $\mathfrak{g}^{\prime}$. As shown above we may assume, up to conjugation, that $G^{\prime}=\operatorname{Aut}\left(g^{\prime}, \eta^{\prime}, \xi, \Phi^{\prime}\right)_{0}$, for $\left(g^{\prime}, \eta^{\prime}, \xi, \Phi^{\prime}\right) \in$ $\mathcal{S}(\xi, \bar{J})$. Let $\overline{\mathfrak{g}}^{\prime}:=\pi\left(\mathfrak{g}^{\prime}\right)=\mathfrak{g}^{\prime} / \xi \subset \mathfrak{h o l}^{T}(\xi, \bar{J})_{0}$. Then $\mathfrak{g}^{\prime}$ has image under $\Upsilon$ given by $\Upsilon_{*} \mathfrak{g}^{\prime}=\overline{\mathfrak{g}}^{\prime} / \overline{\mathfrak{g}}^{\prime} \cap Z\left(\mathfrak{h o r} \mathfrak{r}^{T}(\xi, \bar{J})_{0}\right)$. Since $\Upsilon_{*} \mathfrak{g} \subseteq \mathfrak{h}$ is the Lie algebra of a maximal compact subgroup of $H$, there exists an $h \in H$ so that $\operatorname{Ad}(h)_{*} \Upsilon_{*} \mathfrak{g}^{\prime} \subseteq \Upsilon_{*} \mathfrak{g}$.

Let $\phi \in \operatorname{Fol}\left(M, \mathscr{F}_{\xi}, \bar{J}\right)$ be such that $\Upsilon(\phi)=h$. Then $\hat{\mathfrak{g}}=\operatorname{Ad}(\phi)_{*}\left(\mathfrak{g}^{\prime}\right)$ satisfies $\Upsilon_{*} \hat{\mathfrak{g}} \subseteq \Upsilon_{*} \mathfrak{g}$. Since $\pi(\mathfrak{g})$ contains $Z\left(\mathfrak{h o r}^{T}(\xi, \bar{J})_{0}\right)$, we have $\pi(\hat{\mathfrak{g}}) \subseteq \pi(\mathfrak{g})$. Applying $\phi$ to $\left(g^{\prime}, \eta^{\prime}, \xi, \Phi^{\prime}\right)$ and then a transformation as $f$ above, we get a Sasaki structure $(\tilde{g}, \tilde{\eta}, \xi, \tilde{\Phi})$ with $\operatorname{Aut}(\tilde{g}, \tilde{\eta}, \xi, \tilde{\Phi})_{0}=\tilde{G}$ conjugate to $G^{\prime}$ in $\operatorname{Fol}\left(M, \mathscr{F}_{\xi}, \bar{J}\right)$ and with $\pi(\tilde{\mathfrak{g}}) \subseteq \pi(\mathfrak{g})$.

From Lemma 7 we have $\eta=\tilde{\eta}+d \psi+d^{c} \phi+\alpha$, with $\psi, \phi$ basic and $\alpha \tilde{g}$ harmonic. So $\omega^{T}=\tilde{\omega}^{T}+\frac{1}{2} d d^{c} \phi$. After a gauge transformation (See Remark 8 ) we may assume that $\psi=0$. Any $\bar{X} \in \pi(\tilde{\mathfrak{g}}) \subseteq \pi(\mathfrak{g})$ is both $g^{T}$ and $\tilde{g}^{T}$ Killing. Since

$$
0=\mathcal{L}_{\bar{X}} d d^{c} \phi=d d^{c} \bar{X} \phi,
$$

$\bar{X} \phi$ is constant, and thus $\bar{X} \phi=0$. If $X \in \tilde{\mathfrak{g}}$, then $d(\tilde{\eta}(X))=-X\lrcorner d \tilde{\eta}$. We have

$$
\begin{aligned}
d(\eta(X)) & =d\left(\tilde{\eta}(X)+d^{c} \phi(X)+\alpha(X)\right) \\
& =-X\lrcorner d \tilde{\eta}+d\left(d^{c} \phi(X)\right) \\
& \left.=-X\lrcorner d \tilde{\eta}-(X\lrcorner d d^{c} \phi\right) \\
& =-X\lrcorner d \eta,
\end{aligned}
$$

where the second equality is because $\alpha$ is harmonic and the third because $\mathcal{L}_{X} d^{c} \phi=$ 0 . Therefore $\tilde{\mathfrak{g}} \subseteq \mathfrak{g}$. Since $\tilde{G}$ is maximal, we must have $\tilde{G}=G$. Therefore $G^{\prime}$ is conjugate to $G$ in $\operatorname{Fol}\left(M, \mathscr{F}_{\xi}, \bar{J}\right)$.

Remark 2.17. If we have two Sasaki-extremal structures $\left(g_{i}, \eta_{i}, \xi, \Phi_{i}\right) \in \mathcal{S}(\xi, \bar{J}), i=$ 1,2 , then there is a $\phi \in \operatorname{Fol}\left(M, \mathscr{F}_{\xi}, \bar{J}\right)$ so that $\phi_{*}\left(g_{2}, \eta_{2}, \xi, \Phi_{2}\right)=(\hat{g}, \hat{\eta}, \xi, \hat{\Phi})$ satisfies $\operatorname{Aut}(\hat{g}, \hat{\eta}, \xi, \hat{\Phi})_{0}=\operatorname{Aut}\left(g_{1}, \eta_{1}, \xi, \Phi_{1}\right)_{0}$. One should be able to extend the proof of uniqueness of extremal Kähler metrics $[9$ to the Sasaki case.

From now on $\mathrm{G}^{\prime} \subset \operatorname{Fol}\left(M, \mathscr{F}_{\xi}, \bar{J}\right)$ will be a fixed maximal connected compact subgroup, and $G \subseteq G^{\prime}$ a connected compact subgroup with Lie algebras $\{\xi\} \subseteq \mathfrak{g} \subseteq$ $\mathfrak{g}^{\prime}$. As seen above there is a Sasaki structure $(g, \eta, \xi, \Phi)$ with $G^{\prime}=\operatorname{Aut}(g, \eta, \xi, \Phi)_{0}$. We define several Lie algebras:

$$
\bullet \mathfrak{z}=Z(\mathfrak{g}) \text {, the center of } \mathfrak{g},
$$


- $\mathfrak{z}^{\prime}=C_{\mathfrak{g}^{\prime}}(\mathfrak{g})$, the centralizer of $\mathfrak{g}$ in $\mathfrak{g}^{\prime}$,

- $\mathfrak{z}^{\prime \prime}=C_{\mathfrak{h} \mathfrak{o} l^{T}(\xi, \bar{J})_{0}}(\mathfrak{g})$, the centralizer of $\mathfrak{g}$ in $\mathfrak{h o r}^{T}(\xi, \bar{J})_{0}$,

- $\mathfrak{p}=N_{\mathfrak{g}^{\prime}}(\mathfrak{g})$, the normalizer of $\mathfrak{g}$ in $\mathfrak{g}^{\prime}$,

- $\mathfrak{q}=N_{\mathfrak{h o l} l^{T}(\xi, \bar{J})_{0}}(\mathfrak{g})$, the normalizer of $\mathfrak{g}$ in $\mathfrak{h o l}^{T}(\xi, \bar{J})_{0}$.

We denote by $\mathcal{H}_{g}^{\mathfrak{s}} \subseteq \mathcal{H}_{g}^{b}$ the corresponding space of holomorphy potentials where $\mathfrak{s}$ is one of the above Lie algebras. Note that

$$
\mathcal{H}_{g}^{\mathfrak{z}} \subseteq \mathcal{H}_{g}^{\mathfrak{z}^{\prime}} \subseteq \mathcal{H}_{g}^{\mathfrak{p}} \subseteq \mathcal{H}_{g}^{\mathfrak{g}^{\prime}}
$$

consist of purely imaginary functions, and $\mathcal{H}_{g}^{\mathfrak{z}}$ (respectively $\mathcal{H}_{g}^{\mathfrak{z}^{\prime}}$ ) consist of $G$ invariant functions in $\mathcal{H}_{g}^{\mathfrak{g}}$ (respectively $\mathcal{H}_{g}^{\mathfrak{g}^{\prime}}$ ).

We also have the following whose proof is just as in [25].

Lemma 18. We have the isomorphisms of Lie algebras induced by the injections

$$
\mathfrak{z}^{\prime} / \mathfrak{z} \cong \mathfrak{p} / \mathfrak{g}, \quad \mathfrak{z}^{\prime \prime} / \mathfrak{z} \cong \mathfrak{q} / \mathfrak{g}
$$

\subsection{Relative Futaki invariant.}

2.5.1. Reduced scalar curvature. We let $L_{k}^{2}(M)$ denote the $\mathrm{k}^{\text {th }}$ real Sobolev space. We assume $k>m+1$, where $\operatorname{dim} M=2 m+1$, so that $L_{k}^{2}(M)$ is a Banach algebra. Denote $L_{k, G}^{2}(M)$ to be the subspace of $G$-invariant functions in $L_{k}^{2}(M)$, which is also a Hilbert space and Banach algebra. Note that $L_{k, G}^{2}(M)$ are basic functions since we assume that $\xi \subset \mathfrak{g}$. The $L^{2}$-inner product is defined using the metric of the $G$-invariant Sasaki structure.

We have an orthogonal decomposition

$$
L_{k, G}^{2}(M)=\sqrt{-1} \mathcal{H}_{g}^{\mathfrak{z}} \oplus W_{g, k},
$$

with the projections

$$
\pi_{g}^{G}: L_{k, G}^{2}(M) \rightarrow \sqrt{-1} \mathcal{H}_{g}^{\mathfrak{z}} \text { and } \pi_{g}^{W}: L_{k, G}^{2}(M) \rightarrow W_{g, k} .
$$

Associated to a $G$-invariant Sasaki structure $(g, \eta, \xi, \Phi)$ we define the reduced scalar curvature

$$
s_{g}^{G}=\pi_{g}^{W}\left(s_{g}\right) .
$$

The condition $s_{g}^{G}=0$ is equivalent to $s_{g} \in \sqrt{-1} \mathcal{H}_{g}^{\mathfrak{z}} \subset \mathcal{H}_{g}^{b}$, so this implies that $g$ is Sasaki-extremal.

2.5.2. Reduced Ricci form and Ricci potential. As in 27, 28, we define the reduced Ricci form and Ricci potential. Let $L_{k, G}^{2}\left(\Lambda_{b}^{1,1} M\right)$ be the space of basic $G$-invariant $(1,1)$-forms in $L_{k}^{2}$. As in 28] one can define a projection

$$
\Pi_{g}^{G}: L_{k, G}^{2}\left(\Lambda_{b}^{1,1} M\right) \rightarrow L_{k, G}^{2}\left(\Lambda_{b}^{1,1} M\right),
$$

by

$$
\Pi_{g}^{G} \gamma=\gamma+d d^{c} f
$$

where $f=G_{g}\left(\pi^{W}\left(\omega^{T}, \gamma\right)\right)$. This projection intertwines the trace with $\pi_{g}^{G}$, that is $\left(\omega^{T}, \Pi_{g}^{G} \gamma\right)=\pi_{g}^{G}\left(\omega^{T}, \gamma\right)$.

We obtain the reduced Ricci form 28] by

$$
\rho^{G}=\Pi_{g}^{G} \rho^{T}
$$


and the related identity

$$
\rho^{G}=\rho^{T}+\frac{1}{2} d d^{c} \psi_{g}^{G} .
$$

One has $\psi_{g}^{G}=2 G_{g}\left(\pi^{W}\left(\omega^{T}, \rho^{T}\right)\right)$ and $\rho^{G}=\rho^{T}$ if and only if $s_{g}^{G}=0$.

2.5.3. Relative Futaki invariant. Suppose we have a $G$-invariant Sasaki structure $(g, \eta, \xi, \Phi)$ on $M$. We define the relative Futaki invariant

$$
\mathcal{F}_{G, \xi}(X)=\int_{M} d^{c} \psi_{g}^{G}(X) d \mu_{g},
$$

where $X \in \mathfrak{h o l}^{T}(\xi, \bar{J})$ is any real transversely holomorphic vector field and $\psi_{g}^{G}$ is the Ricci potential (37) of $g$. Though defined in terms of the metric, (38) is independent of the $G$-invariant Sasaki structure in $\mathcal{S}(\xi, \bar{J})$. See [25], and also [5] and [16] for the Sasaki-Futaki invariant, where $\psi_{g}^{G}$ is replaced by the usual Riccipotential $\psi_{g}=2 G_{g}\left(\left(\omega^{T}, \rho^{T}\right)-\left(\omega^{T}, \rho^{T}\right)_{0}\right)$, with

$$
\begin{aligned}
\left(\omega^{T}, \rho^{T}\right)_{0} & =\frac{\int_{M}\left(\omega^{T}, \rho^{T}\right) d \mu_{g}}{\int_{M} d \mu_{g}} \\
& =\frac{\int_{M} s_{g}^{T} d \mu_{g}}{\int_{M} d \mu_{g}} \\
& =\frac{4 m \pi c_{1}\left(\mathscr{F}_{\xi}\right) \cup\left[\omega^{T}\right]^{m-1}}{\left[\omega^{T}\right]^{m}} .
\end{aligned}
$$

the average of the scalar curvature.

In terms of the Hodge decomposition (27) of the dual 1-form $X^{b}=X_{h}^{b}+d u_{X}+$ $d^{c} v_{X}$ we have

$$
\begin{aligned}
\mathcal{F}_{G, \xi}(X) & =\int_{M}\left(J^{*} X_{h}^{b}+J^{*} d u_{X}+J^{*} d^{c} v_{X}, d \psi_{g}^{G}\right) d \mu_{g} \\
& =\int_{M}\left(J^{*} X_{h}^{b}-d^{c} u_{X}+d v_{X}, d \psi_{g}^{G}\right) d \mu_{g} \\
& =\int_{M}\left(d v_{X}, d \psi_{g}^{G}\right) d \mu_{g} \\
& =\int_{M}\left(v_{X}, \Delta_{g} \psi_{g}^{G}\right) d \mu_{g} \\
& =\int_{M} v_{X} s_{g}^{G} d \mu_{g} .
\end{aligned}
$$

The third equality follows because $J^{*} X_{h}^{b}$ is harmonic and $d^{c} u_{X}$ is coclosed.

It follows from (39) that if $X \in \mathfrak{g}$ then $\mathcal{F}_{G, \xi}(X)=0$. Thus we have the $\mathbb{R}$-linear character

$$
\mathcal{F}_{G, \xi}: \mathfrak{q} / \mathfrak{g} \rightarrow \mathbb{R}
$$

A $G$-invariant Sasaki-extremal structure in $\mathcal{S}(\xi, \bar{J})$ has $s_{g}^{G}=0$ if and only if (40) vanishes. 


\section{Deformations of SASAKi Structures}

Besides the transversal Kähler deformations of a Sasaki structure $(g, \eta, \xi, \Phi)$ considered in Lemma 7 we will consider two other deformations. First, we will consider deformations of the transversal complex structure $\bar{J}$ on $\mathscr{F} \xi$. In particular, we will consider deformations equivariant with respect to the compact group $G$. Second, we will also consider deformations of the Reeb vector field $\xi$. Together these give a subspace of the versal deformation space of $(\mathscr{F} \xi, \bar{J})$ as a transversely holomorphic foliation.

\subsection{Deformation of foliations.}

3.1.1. Kuranishi space. We consider the deformations of the transversely holomorphic foliation $\left(\mathscr{F}_{\xi}, \bar{J}\right)$. In particular, we are interested in the deformations of $\left(\mathscr{F}_{\xi}, \bar{J}\right)$ that preserve its structure as a smooth foliation. The existence of a versal space for deformations, which fix the smooth foliation structure, was proved in [12, and the universal property of the versal space was strengthened in [17. Note this requires an assumption on the foliation, of which being transversally Hermitian is sufficient, which is clearly the case for $\left(\mathscr{F}_{\xi}, \bar{J}\right)$.

We denote by $\mathcal{A}^{0, k}=\Gamma\left(\Lambda_{b}^{0, k} \otimes \nu(\mathscr{F})^{1,0}\right)$ the space of smooth basic forms of type $(0, k)$ with values in $\nu(\mathscr{F})^{1,0}$, and we have the Dolbeault complex

$$
0 \rightarrow \mathcal{A}^{0,0} \stackrel{\bar{\partial}_{b}}{\longrightarrow} \mathcal{A}^{0,1} \stackrel{\bar{\partial}_{b}}{\longrightarrow} \cdots
$$

The tangent space to the versal space is the first cohomology of $\left(\mathcal{A}^{0, \bullet}, \bar{\partial}_{b}\right)$ denoted $H_{\bar{\partial}_{b}}^{1}\left(\mathcal{A}^{0, \bullet}\right)$.

The versal space $\mathcal{V}$ is the germ of $\theta^{-1}(0)$ where $\theta$ is an analytic map

$$
H_{\bar{\partial}_{b}}^{1}\left(\mathcal{A}^{0, \bullet}\right) \stackrel{\theta}{\rightarrow} H_{\bar{\partial}_{b}}^{2}\left(\mathcal{A}^{0, \bullet}\right)
$$

Thus there exists a family of transverse holomorphic structures on $\mathscr{F}_{\xi}$ parametrized by $\mathcal{V},\left(\mathscr{F}_{\xi}, \bar{J}_{t}\right)_{t \in \mathcal{V}}$, such that any other deformation is given by a pull-back via a map to $\mathcal{V}$.

As above, we consider a compact group $G$ acting on $\left(\mathscr{F}_{\xi}, \bar{J}\right)$. One can consider the complex of $G$-invariant forms $\mathcal{A}_{G}^{0, k}=\Gamma\left(\Lambda_{b}^{0, k} \otimes \nu(\mathscr{F})^{1,0}\right)^{G}$,

$$
0 \rightarrow \mathcal{A}_{G}^{0,0} \stackrel{\bar{\partial}_{b}}{\longrightarrow} \mathcal{A}_{G}^{0,1} \stackrel{\bar{\partial}_{b}}{\longrightarrow} \cdots
$$

By considering Hodge theory for transversally elliptic operators one can show that the cohomology of (43) is naturally identified with $H_{\bar{\partial}_{b}}^{k}\left(\mathcal{A}^{0, \bullet}\right)^{G}$, the cohomology classes fixed by $G$ of (41). The tangent space of the subspace $\mathcal{V}^{G} \subseteq \mathcal{V}$ of $G$ equivariant deformations is $H_{\bar{\partial}_{b}}^{1}\left(\mathcal{A}^{0, \bullet}\right)^{G}$.

3.1.2. Sasaki structures. Suppose we have a family of $G$-invariant transversal complex structures $\left(\mathscr{F}_{\xi}, \bar{J}_{t}\right), t \in \mathcal{B} \subseteq \mathcal{V}^{G}$, with $\mathcal{B}$ a smooth subspace. By the results of [11, which extend the stability result of Kodaira and Spencer on deformations of Kähler manifolds to deformations of foliations fixing the differentiable structure, we have transverse Kähler structures $\omega_{t}^{T}$ on $\left(\mathscr{F}_{\xi}, \bar{J}_{t}\right)$ with $t, \in \mathcal{B}$, after possibly shrinking $\mathcal{B}$. Although the transversely Kähler property is stable, there is a further obstruction to the existence of a Sasaki structure compatible with $\left(\mathscr{F}_{\xi}, \bar{J}_{t}\right)$ for $t \in \mathcal{B}$. The necessary and sufficient condition for the existence of a Sasaki structure were obtained in [22] for more general deformations, not necessarily preserving the 
smooth structure of $\mathscr{F}$. But for our purposes, we will only consider deformations of $\left(\mathscr{F}_{\xi}, \bar{J}\right)$ preserving the smooth foliation structure.

Definition 19. A deformation $\left(\mathscr{F}_{\xi}, \bar{J}_{t}\right), t \in \mathcal{B} \subseteq \mathcal{V}$ of the underlying foliation of a Sasaki structure $(g, \eta, \xi, \Phi)$ is of $(1,1)$-type if for all $t \in \mathcal{B}$ the $(0,2)$-component of the Euler class $\left[d \eta^{0,2}\right] \in H_{b, t}^{0,2}\left(M / \mathscr{F}_{\xi}\right)$ is zero, where $H_{b, t}^{0,2}\left(M / \mathscr{F}_{\xi}\right)=H_{\bar{\partial}_{b, t}}^{2}\left(\Gamma\left(\Lambda_{b}^{0, \bullet}\right)\right)$ is the basic Dolbeault cohomology for the transversal complex structure $\bar{J}_{t}$.

Theorem $20([22])$. Let $\left(\mathscr{F}_{\xi}, \bar{J}_{t}\right), t \in \mathcal{B} \subseteq \mathcal{V}$ be a deformation of the Reeb foliation of $(g, \eta, \xi, \Phi)$. Then there exists a smooth family of $\left(g_{t}, \eta_{t}, \xi, \Phi_{t}\right) \in \mathcal{S}\left(\xi, \bar{J}_{t}\right), t \in V \subset$ $\mathcal{B}$ of compatible Sasaki structures, where $V$ is a neighborhood of zero in $\mathcal{B}$, if and only if the deformation is of $(1,1)$-type restricted to $V$.

An application of a transversal Kodaira-Nakano vanishing theorem gives the following which is basically Corollary 1.4 of $[22]$.

Proposition 21. Let $\left(\mathscr{F}_{\xi}, \bar{J}_{t}\right), t \in \mathcal{B}$ be a deformation of the underlying foliation of a Sasaki structure $(g, \eta, \xi, \Phi)$, and suppose the first Chern class $c_{1}^{b}\left(\mathscr{F}_{\xi}\right)=\frac{1}{2 \pi}\left[\rho^{T}\right] \in$ $H_{b}^{2}\left(M / \mathscr{F}_{\xi}, \mathbb{R}\right)$ is representable by a basic positive $(1,1)$-form, then after restricting to a neighborhood of zero $V \subset \mathcal{B}$ the deformation is of $(1,1)$-type.

Remark 3.22. In particular, the proposition is applicable if $(g, \eta, \xi, \Phi)$ is SasakiEinstein or more generally $\operatorname{Ric}^{T}>0$. But in order to apply Kodaira-Nakano vanishing to prove $H_{b, t}^{0,2}\left(M / \mathscr{F}_{\xi}\right)$ for $t \in V$ we only need that the transverse anti-canonical bundle $\bigwedge^{m, 0} \nu\left(\mathscr{F}_{\xi}\right)$ is positive.

Example 3.23 Let $Z_{a}=\mathbb{C}^{2} / \Lambda$ be the complex torus given by the lattice $\Lambda=$ $\mathbb{Z}\left\{\lambda_{1}, \ldots, \lambda_{4}\right\} \subset \mathbb{C}^{2}$ with $\lambda_{1}=(1,0), \lambda_{2}=(0,1), \lambda_{3}=(i, 0), \lambda_{4}=(a, i)$ where $a \in \mathbb{C}$. Let $x_{1}, \ldots, x_{4}$ be dual real coordinates to the lattice vectors $\lambda_{1}, \ldots, \lambda_{4}$. Then

$$
\omega=d x_{1} \wedge d x_{3}+d x_{2} \wedge d x_{4}
$$

is integral, $[\omega] \in H^{2}(Z, \mathbb{Z})$, and so defines a smooth $S^{1}$ bundle $\mathbf{L}$ with total space $M$. Let $z_{1}, z_{2}$ be the standard holomorphic coordinates on $\mathbb{C}^{2}$. Then a routine calculation gives

$\omega=\frac{i}{2} d z_{1} \wedge d \bar{z}_{1}+\frac{i}{2} d z_{2} \wedge d \bar{z}_{2}+\frac{a}{4} d \bar{z}_{1} \wedge d z_{2}+\frac{\bar{a}}{4} d z_{1} \wedge d \bar{z}_{2}-\frac{\bar{a}}{4} d z_{1} \wedge d z_{2}-\frac{a}{4} d \bar{z}_{1} \wedge d \bar{z}_{2}$ and $\left[\omega^{0,2}\right]=-\frac{a}{4}\left[d \bar{z}_{1} \wedge d \bar{z}_{2}\right]$ is nonzero in $H^{0,2}(Z)$ for $a \neq 0$. When $a=0$ as a $\mathbb{C}$-bundle $\mathbf{L}$ has a natural holomorphic structure and polarizes $Z_{0}$, and $M$ has a natural Sasaki structure with transversal Kähler form $\omega$ and Reeb foliation $\mathscr{F}_{\xi}$ given by the $S^{1}$ bundle $\mathbf{L}$ with leaf space $Z_{0}$. But for $a \neq 0$ there is no complex structure on the $\mathbb{C}$-bundle $\mathbf{L}$ and no compatible Sasaki structure on $M$ with $\mathscr{F} \xi$ given by $\mathbf{L}$ with leaf space $Z_{a}$.

In fact, one can prove that for $a \in \mathbb{C} \backslash \mathbb{Q}+i \mathbb{Q}$ there is no integral nondegenerate $(1,1)$-form on $Z_{a}$. Thus $Z_{a}$ is not algebraic for $a \in \mathbb{C} \backslash \mathbb{Q}+i \mathbb{Q}$.

If we have a $G$-equivariant deformation of $(1,1)$-type $\left(\mathscr{F}_{\xi}, \bar{J}_{t}\right), t \in \mathcal{B} \subseteq \mathcal{V}^{G}$, then the family $\left(g_{t}, \eta_{t}, \xi, \Phi_{t}\right), t \in \mathcal{B}$ of Theorem 20 can be taken to be $G$-invariant by averaging $\eta_{t}$ by the $G$-action. In the following we will assume the deformed structures $\left(g_{t}, \eta_{t}, \xi, \Phi_{t}\right)$ are $G$-invariant. 


\subsection{Sasaki cone.}

3.2.1. Deforming the Reeb vector field in the Sasaki cone. We have a $G$-invariant Sasaki structure $(g, \eta, \xi, \Phi)$, where $G$ has Lie algebra $\mathfrak{g}$ with center $\mathfrak{z}$ with $\xi \in \mathfrak{z}$.

Definition 24. We define the Sasaki cone of $\mathfrak{z} \subseteq \mathfrak{a} \mathfrak{u}(g, \eta, \xi, \Phi)$ to be $\mathfrak{z}^{+}=\{\zeta \in \mathfrak{z}$ : $\eta(\zeta)>0\}$, which is clearly open in $\mathfrak{z}$.

If $\zeta \in \mathfrak{z}^{+}$, then $\eta_{\zeta}=\eta(\zeta)^{-1} \eta$ is a contact form for $D=\operatorname{ker} \eta$ with Reeb vector field $\zeta$. It follows from Proposition 12 that $\left(g_{\zeta}, \eta_{\zeta}, \zeta, \Phi_{\zeta}\right)$ is a Sasaki structure with the same underlying $\mathrm{CR}$ structure, where $\Phi_{\zeta}(X)=\Phi(X)-\eta_{\zeta}(X) \Phi(\zeta)$ and $g_{\zeta}$ is defined in (11).

Let $T^{r} \subseteq G$ be the connected component of the identity of the center. We get an alternative description of $\mathfrak{z}^{+}$if we consider the moment map for the Hamiltonian action on the cone $C(M)=\mathbb{R}_{>0} \times M$. In fact the moment map for the symplectic action of $T^{r}$ on $C(M)$ is given in terms of the contact form by

$$
\mu_{\eta}: C(M) \rightarrow \mathfrak{z}^{*},
$$

where $\mu_{\eta}(x, r)(X)=r^{2} \eta_{x}(X)$ with $X \in \mathfrak{z}$ also denoting the vector field induced on $C(M)$. The image of (44) is a strongly convex rational polyhedral cone $\mathcal{C}_{\mathfrak{z}}^{*} \subset \mathfrak{z}^{*}$ ([10]). Although the map $\mu_{\eta}$ depends on the contact form $\eta$, the image $\mathcal{C}_{\mathfrak{z}}^{*}$ is independent of transversal Kähler deformations, considered in Lemma 7, and the deformations of the Reeb vector field $\xi \in \mathfrak{z}^{+}$considered above.

By Farkas' theorem, the dual cone $\mathcal{C}_{\mathfrak{z}}$ to $\mathcal{C}_{\mathfrak{z}}^{*}$ is also a strongly convex polyhedral cone. From the definition of $\mu_{\eta}$ we see that

$$
\mathfrak{z}^{+}=\stackrel{\circ}{\mathcal{C}}_{\mathfrak{z}} .
$$

We will consider deformations of the Sasaki structures $\left(g_{t}, \eta_{t}, \xi, \Phi_{t}\right) \in \mathcal{S}\left(\xi, \bar{J}_{t}\right), t \in$ $\mathcal{B}$ of the previous section. Given $\phi \in L_{k, G}^{2}(M)$, with $k>m+5$, and $\xi_{\alpha}=\xi+\alpha \in \mathfrak{z}^{+}$ we consider the Sasaki structure $\left(g_{t, \alpha, \phi}, \eta_{t, \alpha, \phi}, \xi_{\alpha}, \Phi_{t, \alpha, \phi}\right) \in \mathcal{S}\left(\xi_{\alpha}, \bar{J}_{t}\right)$ with

$$
\begin{aligned}
\eta_{t, \alpha, \phi} & =\eta_{t, \xi_{\alpha}}+d^{c} \phi, \\
\Phi_{t, \alpha, \phi} & =\Phi_{t, \xi_{\alpha}}-\left(\xi_{\alpha} \otimes\left(\eta_{t, \xi_{\alpha}, \phi}-\eta_{t, \xi_{\alpha}}\right) \circ \Phi_{t, \xi_{\alpha}},\right.
\end{aligned}
$$

and $g_{t, \alpha, \phi}$ defined from (46) and (47) as in (11). Therefore we have a space of Sasaki structures parametrized by $(t, \alpha, \phi) \in \mathcal{B} \times \mathfrak{z} \times L_{k, G}^{2}(M)$, in a neighborhood of zero. The restriction $k>m+5$ ensures that the curvature tensors of $g_{t, \alpha, \phi}$ are well defined.

3.2.2. Nondegeneracy of the relative Futaki invariant. As the notation in (38) suggests the dependence of $\mathcal{F}_{G, \xi_{\alpha}}$ on $\xi_{\alpha}=\xi+\alpha \in \mathfrak{z}^{+}$will be important. In a following section we will compute the derivative

$$
D_{g} \mathcal{F}_{G, \xi+t \alpha}(\alpha)=\left.\frac{d}{d t} \mathcal{F}_{G, \xi+t \alpha}\right|_{t=0} .
$$

Note that one must be careful that as $\xi_{\alpha}$ varies in $\mathfrak{z}^{+}$one cannot assume that $\mathfrak{h o r}^{T}\left(\xi_{\alpha}, \bar{J}\right)$ is unchanged because we are changing the foliation. We assume that our starting structure $(g, \eta, \xi, \Phi)$ has $G^{\prime}=\operatorname{Aut}(g, \eta, \xi, \Phi)_{0}$ a maximal compact subgroup of $\operatorname{Fol}\left(\mathscr{F}_{\xi}, \bar{J}\right)$. We restrict $\mathcal{F}_{G, \xi_{\alpha}}$ to $\mathfrak{p} / \mathfrak{g}$ and differentiate with respect to $\alpha \in \mathfrak{z}$ at $\alpha=0$ to get

$$
D_{g} \mathcal{F}_{G, \xi}: \mathfrak{p} / \mathfrak{g} \cong \mathfrak{z}^{\prime} / \mathfrak{z} \rightarrow \mathfrak{z}^{*}
$$


Definition 25. The Futaki invariant relative to $G$ is said to be $G^{\prime}$-nondegenerate if 48) is injective.

\section{Proof of MAin theorem}

The proof of the main theorem will depend on variation formulae as we vary the Sasaki structure as in (46).

For $X \in \mathfrak{h o r}^{T}(\xi, \bar{J})_{0}^{1,0}$ the normalized potential $f_{X} \in \mathcal{H}_{g}^{b}$ can be written in terms of the Green's function $G_{g}$

$$
\left.f_{X}=-\sqrt{-1} G_{g}\left(\bar{\partial}_{b}^{*}(X\lrcorner d \eta\right)\right),
$$

though this will not be used.

4.1. Variation formulae. We will consider the first order variations of holomorphy potentials and the reduced scalar curvature with respect to varying the Sasaki structure in (46), more precisely, the derivative with respect to $\phi \in L_{k, G}^{2}(M)$ or $\alpha \in \mathfrak{z}$. In the following $D_{g}$ will denote the derivative with respect to variations of the Sasaki structure at $(g, \eta, \xi, \Phi)$. The proof of the first lemma is easy.

Lemma 26. Let $X \in \mathfrak{h o r}^{T}(\xi, \bar{J})_{0}^{1,0}$ with holomorphy potential $f_{X}=u_{x}+\sqrt{-1} v_{X}$. Then

$$
D_{g} f_{X}(\phi)=X \phi
$$

and if $X=V-\sqrt{-1} \bar{J} V$ real components are $D_{g} u_{X}(\phi)=V \phi, D_{g} v_{X}(\phi)=-\bar{J} V \phi$.

If $\bar{X}=\bar{V}-\sqrt{-1} \bar{J} \bar{V}$ with $V \in \mathfrak{g}^{\prime}$ and $\alpha \in C_{\mathfrak{g}^{\prime}}(X)$, then a non-normalized holomorphy potential is $f_{X}=\sqrt{-1} \eta(V)$. And

$$
D_{g} f_{X}(\alpha)=-\sqrt{-1} \eta(\alpha) \eta(V) .
$$

Lemma 27. Let $(g, \eta, \xi, \Phi)$ be a $G$-invariant Sasaki structure. The variation of $s_{g}^{G}$ in the direction $\phi \in L_{k, G}^{2}(M)$ is

$$
D_{g} s_{g}^{G}(\phi)=-2 \mathbb{L}_{g} \phi+\left(d \phi, d s_{g}^{G}\right) .
$$

If $s_{g}^{G}=0$, then the variation of $s_{g}^{G}$ in the direction $\alpha \in C_{\mathfrak{g}^{\prime}}(\mathfrak{g})$ is given by

$$
D_{g} s_{g}^{G}(\alpha)=\left(\mathbb{1}-\pi_{g}^{G}\right)\left(\eta(\alpha)\left(2 s_{g}-s_{0}+2 m\right)-2(m+1) \Delta_{b} \eta(\alpha)\right)
$$

Proof. The formula (49) was proved in [28. More precisely, it is proved that

$$
\left.D_{g}\left(\pi^{G} s^{G}\right)(\phi)=\partial_{g}^{\#}\left(\pi_{g}^{G} s_{g}\right)\right\lrcorner \partial \phi=\frac{1}{2}\left(d \phi, d\left(\pi_{g}^{G} s_{g}\right)\right)
$$

from which (49) follows.

For (50) we consider the variation of Sasaki structures $\xi_{t}=\xi+t \alpha$ with $\alpha \in C_{\mathfrak{g}^{\prime}}(\xi)$, $\eta_{t}=\eta\left(\xi_{t}\right)^{-1} \eta$, and fixing the CR structure. Let $f_{t}=\eta\left(\xi_{t}\right)^{-1}$, then $\left.\omega_{t}^{T}\right|_{D}=$ $\left.f_{t} \omega^{T}\right|_{D}$, which is just a conformal change. But the calculation of the variation of the curvature is more subtle as the foliation with respect to which the transverse connection, ${ }^{t} \nabla^{T}$, is defined is changing. One calculates, with $X, Y \in \Gamma(D)$

$$
\begin{aligned}
{ }^{t} \nabla_{X}^{T} Y=\nabla_{X}^{T} Y & +\frac{1}{2}\left(d \log f(X) Y+d \log f(Y) X-g^{T}(X, Y)(d \log f)^{\#}\right) \\
& +f\left(g^{T}(\Phi(X), Y) \pi_{\xi}\left(\xi_{t}\right)-g\left(\xi_{t}, Y\right) \Phi(X)-g\left(\xi_{t}, X\right) \Phi(Y)\right),
\end{aligned}
$$


where $\pi_{\xi}: T M \rightarrow D$ is the orthogonal projection with respect to $g$ and $f=f_{t}$. And

$$
\begin{aligned}
\dot{\nabla}_{X}^{T} Y=\left.\frac{d}{d t}{ }^{t} \nabla_{X}^{T} Y\right|_{t=0}= & \nabla_{X}^{T} Y+\frac{1}{2}\left(d \dot{f}(X) Y+d \dot{f}(Y) X-g^{T}(X, Y)(d \dot{f})^{\#}\right) \\
& \left.+g^{T}(\Phi(X), Y) \pi_{\xi}(\alpha)-g(\alpha, Y) \Phi(X)-g(\alpha, X) \Phi(Y)\right) .
\end{aligned}
$$

Using (52) one calculates, via a long but routine calculation the derivative at $t=0$ of the transverse curvature $\dot{R}^{T}(X, Y) Z$. And contracting the result gives

$$
\begin{aligned}
\left.\frac{d}{d t} s_{t}^{T}\right|_{t=0} & =-\dot{f} s_{g}^{T}+2(m+1) \Delta_{b} \dot{f} \\
& =\eta(\alpha)\left(s_{g}+2 m\right)-2(m+1) \Delta_{B} \eta(\alpha) .
\end{aligned}
$$

It remains to differentiate $\pi_{g}^{G}$. We let $\pi_{t}^{G}: L_{k, G}^{2}(M) \rightarrow \sqrt{-1} \mathcal{H}_{g_{t}}^{\mathfrak{z}} \subset L_{k, G}^{2}(M)$ be the projection defined by the above Sasaki structure with $\xi_{t}=\xi+t \alpha$ and fixed CR structure. Since $s_{g}^{G}=\left(\mathbb{1}-\pi_{g}^{G}\right) s_{g}=0$, it is sufficient to compute $(\mathbb{1}-$ $\left.\pi_{g}^{G}\right)\left(\left.\frac{d}{d t} \pi_{t}^{G}\right|_{t=0}\right) s_{g}$. We claim that

$$
\left(\mathbb{1}-\pi_{g}^{G}\right)\left(\left.\frac{d}{d t} \pi_{t}^{G}\right|_{t=0}\right) s_{g}=\left(\mathbb{1}-\pi_{g}^{G}\right)\left(-\eta(\alpha)\left(s_{g}-s_{0}\right)\right) .
$$

We may assume that $\partial_{g}^{\#} s_{g} \not \equiv 0$, otherwise both sides vanish. Let $\left\{X_{0}=\xi, X_{1}, \ldots, X_{r}\right\}$ be a basis of $\mathfrak{z}$ with $X_{1}$ chosen so that $\bar{J} \bar{X}_{1}+\sqrt{-1} \bar{X}_{1}=\partial_{g}^{\#} s_{g}$ and $\eta\left(X_{1}\right)=s_{g}-s_{0}$. Then $p_{t}^{0}=1, p_{t}^{1}=\eta_{t}\left(X_{1}\right), \ldots, p_{t}^{r}=\eta_{t}\left(X_{r}\right)$ is a basis of $\mathcal{H}_{g_{t}}^{z}$ for small $t$. By the Gramm-Schmidt procedure, using the $L^{2}$ inner product induced by $g$, we obtain an orthonormal basis $\left\{f_{t}^{0}, \ldots, f_{t}^{r}\right\}$ from $\left\{p_{t}^{j}\right\}$. In terms of this basis we have $\pi_{t}^{G} s_{g}=\sum_{j=0}^{r}\left\langle f_{t}^{j}, s\right\rangle_{L^{2}} f_{t}^{j}$. Thus we have

$$
\left(\mathbb{1}-\pi_{g}^{G}\right)\left(\left.\frac{d}{d t} \pi_{t}^{G}\right|_{t=0}\right) s_{g}=\left.\left(\mathbb{1}-\pi_{g}^{G}\right) \sum_{j=0}^{r}\left\langle f_{0}^{j}, s\right\rangle_{L^{2}} \frac{d}{d t} f_{t}^{j}\right|_{t=0},
$$

because each $f_{0}^{j}$ is in the kernel of $\left(\mathbb{1}-\pi_{g}^{G}\right)$.

Note that only the $j=0,1$ terms in (55) are possibly non-trivial. We have $p_{t}^{1}=\eta(\xi+t \alpha)^{-1} \eta\left(X_{1}\right)=\eta(\xi+t \alpha)^{-1}\left(s_{g}-s_{0}\right)$, and $f_{t}^{0}=\left(\operatorname{Vol}\left(g_{t}\right)\right)^{-1 / 2}$. So we have

$$
f_{t}^{1}=\frac{p_{t}^{1}-\left\langle p_{t}^{1}, f_{t}^{0}\right\rangle_{L^{2}} f_{t}^{0}}{\left\|p_{t}^{1}-\left\langle p_{t}^{1}, f_{t}^{0}\right\rangle_{L^{2}} f_{t}^{0}\right\|_{L^{2}}} .
$$

Since $\left.\frac{d}{d t} f_{t}^{0}\right|_{t=0}$ is a constant function, (55) is

$$
\left(\mathbb{1}-\pi_{g}^{G}\right)\left(\left.\frac{d}{d t} \pi_{t}^{G}\right|_{t=0}\right) s_{g}=\left.\left\|s_{g}-s_{0}\right\|_{L^{2}}\left(\mathbb{1}-\pi_{g}^{G}\right) \frac{d}{d t} f_{t}^{1}\right|_{t=0} .
$$

Again, because constant functions and $p_{0}^{1}$ are annihilated by $\left(\mathbb{1}-\pi_{g}^{G}\right)$,

$$
\left.\frac{d}{d t} f_{t}^{1}\right|_{t=0}=\frac{-\eta(\alpha)\left(s_{g}-s_{0}\right)}{\left\|s_{g}-s_{0}\right\|_{L^{2}}} \quad \bmod \quad \operatorname{ker}\left(\mathbb{1}-\pi_{g}^{G}\right)
$$

and (54) follows.

Lemma 27 and (39) has the following consequence 
Proposition 28. Suppose $(g, \eta, \xi, \Phi)$ be a $G$-invariant Sasaki structure satisfying $s_{g}^{G}=0$. Then the derivative of the relative Futaki invariant is

$$
D_{g} \mathcal{F}_{G, \xi, X}(\alpha)=\int_{M} v_{X}\left(\mathbb{1}-\pi_{g}^{G}\right)\left(\eta(\alpha)\left(2 s_{g}-s_{0}+2 m\right)-2(m+1) \Delta_{b} \eta(\alpha)\right) d \mu_{g},
$$

where $X \in \mathfrak{z}^{\prime}$ has potential $\sqrt{-1} v_{X}$ and $\alpha \in \mathfrak{z}$.

This has the following consequence.

Corollary 29. Let $(g, \eta, \xi, \Phi)$ be a $G$-invariant Sasaki-Einstein structure. Then if $\mathfrak{p} / \mathfrak{g} \cong \mathfrak{z}^{\prime} / \mathfrak{z}$ nonzero, the relative Futaki invariant is degenerate.

Proof. By the Lichnerowicz-Matsushima theorem the Killing potentials $\eta(\alpha)$, when normalized to have zero integral, $v_{\alpha}=\eta(\alpha)-\eta(\alpha)_{0}$ satisfy $\Delta_{b} v_{\alpha}=4(m+1) v_{\alpha}$. It is then easy to see that the integrand in Proposition 28 vanishes.

\subsection{Main theorem.}

4.2.1. Proof of main theorem. A Sasaki metric $g_{t, \alpha, \phi}$ as in (46) with $\phi \in L_{k+4, G}^{2}(M)$, $k>m+1$, is $G$-invariant. We have the space of holomorphy potentials $\mathcal{H}_{t, \alpha, \phi}^{\mathfrak{g}}$ for $g_{t, \alpha, \phi}$, and the subspace of $G$-invariant potentials $\mathcal{H}_{t, \alpha, \phi}^{\mathfrak{z}} \subseteq \mathcal{H}_{t, \alpha, \phi}^{\mathfrak{g}}$. Using the metric $g_{t, \alpha, \phi}$ to define the $L^{2}$ inner product on $\phi \in L_{k, G}^{2}(M)$ we have the orthogonal decomposition

and the projections

$$
L_{k, G}^{2}(M)=\sqrt{-1} \mathcal{H}_{t, \alpha, \phi}^{\mathfrak{z}} \oplus W_{k, t, \alpha, \phi},
$$

$$
\pi_{t, \alpha, \phi}^{G}: L_{k, G}^{2}(M) \rightarrow \sqrt{-1} \mathcal{H}_{t, \alpha, \phi}^{\mathfrak{z}}, \text { and } \pi_{t, \alpha, \phi}^{W}: L_{k, G}^{2}(M) \rightarrow W_{k, t, \alpha, \phi} .
$$

The reduced scalar curvature of $g_{t, \alpha, \phi}$ is given by

$$
s_{t, \alpha, \phi}^{G}=\pi_{t, \alpha, \phi}^{W}\left(s_{t, \alpha, \phi}\right)=\left(\mathbb{1}-\pi_{t, \alpha, \phi}^{G}\right)\left(s_{t, \alpha, \phi}\right)
$$

We are looking for solutions of the equation

$$
s_{t, \alpha, \phi}^{G}=0 .
$$

For $(t, \alpha, \phi) \in \mathcal{U} \subset \mathcal{B} \times \mathfrak{z} \times L_{k+4, G}^{2}(M)$ with $\mathcal{U}$ a small enough neighborhood of $(0,0,0)$ the associated Sasaki structure (46) is well defined and $\pi_{0}^{W}: W_{k, t, \alpha, \phi} \rightarrow$ $W_{k, o}$ is an isomorphism.

Let $\mathcal{V}=\mathfrak{U} \cap \mathcal{B} \times \mathfrak{z} \times W_{k+4,0}$. Then we define a map

$$
\begin{array}{ccc}
\mathscr{S}: \mathcal{V} & \rightarrow \mathcal{B} \times W_{k, 0} \\
(t, \alpha, \phi) & \mapsto\left(t, \pi_{0}^{W}\left(s_{t, \alpha, \phi}^{G}\right)\right)
\end{array}
$$

Lemma 30. The map $\mathscr{S}$ is $C^{1}$ and its differential is Fredholm. Assume that the Sasaki structure $(g, \eta, \xi, \Phi)$ at $(0,0,0) \in \mathcal{V}$ has vanishing reduced scalar curvature $s_{g}^{G}=0$, then the differential of $\mathscr{S}$ at $(t, \alpha, \phi)=(0,0,0)$ is

$$
\left[\begin{array}{lll}
\mathbb{1} & 0 & 0 \\
* & \mathcal{S}_{g}^{G}
\end{array}\right]
$$

where

$$
\begin{aligned}
\mathcal{S}_{g}^{G}(\dot{\alpha}, \dot{\phi}) & \left.=-2 \mathbb{L}_{g} \dot{\phi}+\pi_{0}^{W}\left(\dot{s}_{g}^{G}\right)(\alpha)\right) \\
& \left.=-2 \mathbb{L}_{g} \dot{\phi}+\pi_{0}^{W}\left(\eta(\alpha)\left(2 s_{g}-s_{0}+2 m\right)-2(m+1) \Delta_{b} \eta(\alpha)\right)\right) .
\end{aligned}
$$


Proof. Since the reduced scalar curvature $s_{t, \alpha, \phi}^{G}$ is $C^{1}$ in $(t, \alpha, \phi)$, the map $\mathscr{S}$ is $C^{1}$. The formula for $\mathcal{S}_{g}^{G}(\dot{\alpha}, \dot{\phi})$ follows from Lemma 27.

Proposition 31. Suppose that $(g, \eta, \xi, \Phi)$ at $(0,0,0) \in \mathcal{V}$ had vanishing reduced scalar curvature $s_{g}^{G}=0$, then the Fréchet derivative of $\mathscr{S}$, defined in (59), at $(t, \alpha, \phi)=(0,0,0)$ has index $\operatorname{dim} \mathfrak{z}$ and is an submersion if and only if the relative Futaki invariant $\mathcal{F}_{G, \xi}$ is nondegenerate at $\xi$.

Proof. Note that $D_{g} \mathscr{S}$ is a compact perturbation of

$$
\mathcal{B} \times \mathfrak{z} \times W_{k+4,0} \ni(s, \dot{\alpha}, \dot{\phi}) \mapsto\left(s,-2 \llbracket_{g} \dot{\phi}\right) \in \mathcal{B} \times W_{k, 0}
$$

Since the index of $\mathbb{L}_{g}: W_{k+4,0} \rightarrow W_{k, 0}$ is zero, the index of $D_{g} \mathscr{S}$ must be $\operatorname{dim} \zeta$.

If $D_{g} \mathscr{S}$ is not surjective, there is a $\psi \in W_{k, 0}$ in the cokernel. We have from (60)

$$
\left.\left\langle\mathbb{L}_{g} \dot{\phi}, \psi\right\rangle=0, \quad \text { and }\left\langle\pi_{0}^{W}\left(\dot{s}_{g}^{G}\right)(\alpha)\right), \psi\right\rangle=0,
$$

for all $\dot{\phi} \in W_{k+4,0}$. The first equation implies $\psi \in \sqrt{-1} \mathcal{H}_{g}^{\mathfrak{z}^{\prime}}$, so $X=\bar{J} \operatorname{grad} \psi \in \mathfrak{z}^{\prime}$. Thus

$$
\int_{M} \psi \pi_{0}^{W}\left(\dot{s}_{g}^{G}(\dot{\alpha})\right) d \mu_{g}=\int_{M} \psi \dot{s}_{g}^{G}(\dot{\alpha}) d \mu_{g}=D_{g} \mathfrak{F}_{G, \xi, X}(\dot{\alpha}),
$$

where the second equality uses (39) and that $s_{g}^{G}=0$. If $\mathcal{F}_{G, \xi}$ is nondegenerate, then this implies $X \in \mathfrak{z}$ and $\psi \in \sqrt{-1} \mathcal{H}_{g}^{\mathfrak{z}}$ contradicting $\psi \in W_{k, 0}$.

Theorem 32. Let $\left(\mathscr{F}_{\xi}, \bar{J}_{t}\right), t \in \mathcal{B}$, be a $G$-equivariant $(1,1)$-type deformation with $\mathcal{B}$ smooth and fixing the smooth structure of $\mathscr{F}_{\xi}$, where $G$ is a compact connected group with $\xi \in \mathfrak{g}$. Suppose $(g, \eta, \xi, \Phi) \in \mathcal{S}\left(\xi, \bar{J}_{0}\right)$ has vanishing reduced scalar curvature $s_{g}^{G}=0$. If the relative Futaki invariant $\mathcal{F}_{G, \xi}$ is nondegenerate at $\xi$, then there is a neighborhood $\mathcal{V}$ of $(0,0,0) \in \mathcal{B} \times \mathfrak{z} \times C_{b}^{\infty}(M)^{G}$ so that

$$
\mathcal{E}=\left\{(t, \alpha, \phi) \in \mathcal{V}:\left(g_{t, \alpha, \phi}, \eta_{t, \alpha, \phi}, \xi+\alpha, \Phi_{t, \alpha, \phi}\right) \text { has } s_{t, \alpha, \phi}^{G}=0\right\},
$$

is a smooth manifold of dimension $\operatorname{dim}_{R} \mathcal{B}+\operatorname{dim}_{R} \mathfrak{z}$.

Furthermore, the map $\varpi: \mathcal{E} \rightarrow \mathcal{B}, \varpi(t, \alpha, \phi)=t$ is a submersion with fibers of dimension $\operatorname{dim}_{R} \mathfrak{z}$. And any $\left.(t, \alpha, \phi)\right) \in \mathcal{E}$ has $\phi \in C_{b}^{\infty}(M)^{G}$.

Proof. By Proposition 31 the map (60) is a submersion at $(0,0,0)$. Let $K=$ ker $D_{g} \mathscr{S} \subset T_{0} \mathcal{B} \times \mathfrak{z} \times W_{k+4,0}$. We identify $\mathcal{B} \subset T_{0} \mathcal{B}$ as a Euclidean space. Let $\pi: \mathcal{V}=\mathcal{U} \cap \mathcal{B} \times \mathfrak{z} \times W_{k+4,0} \rightarrow K$ be the orthogonal projection. Then the differential at zero of

$$
\mathscr{S} \times \pi: \mathcal{V} \rightarrow \mathcal{B} \times W_{k, 0} \times K
$$

is an isomorphism. The inverse function theorem provides an inverse, and $\mathcal{B} \times K \ni$ $(t, s) \mapsto(\mathscr{S} \times \pi)^{-1}(t, 0, s)$, parametrizes $\mathcal{E}$.

$\varpi$ is a submersion because $S$ is orthogonal to $\mathcal{B} \times\{0\} \times\{0\}$. If $(t, \alpha, \phi) \in \mathcal{E}$, then $g_{t, \alpha, \phi}^{T}$ is transversely extremal. The regularity result of [20], applied in a local foliation chart, shows that $\phi \in C_{b}^{\infty}(M)^{G}$.

4.2.2. Maximal torus case. The case in which $G=T^{r} \subseteq G^{\prime}$ is a maximal torus gives a somewhat stronger result than in general. Furthermore, it is easier to find examples, because the nondegeneracy of the Futaki invariant holds trivially. In this section $G=T^{r}$ is a maximal torus in the maximal compact subgroup $G^{\prime} \subset \operatorname{Fol}\left(M, \mathscr{F}_{\xi}, \bar{J}\right)$. Note that we have $\mathfrak{z}=\mathfrak{z}^{\prime}=\mathfrak{g}$.

The proof of the following is obvious. 
Lemma 33. Suppose $G^{\prime} \subset \operatorname{Fol}(M, \mathscr{F} \xi, \bar{J})$ is maximal compact and $G=T^{r} \subseteq G^{\prime}$ is a maximal torus. Then $\mathfrak{p} / \mathfrak{g}=\mathfrak{z}^{\prime} / \mathfrak{z}=0$.

Corollary 34. Let $\left(\mathscr{F}_{\xi}, \bar{J}_{t}\right), t \in \mathcal{B}$, be a $G$-equivariant deformation of $(1,1)$-type with $\mathcal{B}$ smooth and fixing the smooth structure of $\mathscr{F}_{\xi}$, where $G=T^{r}$ is a maximal torus of $G^{\prime}=\operatorname{Aut}(g, \eta, \xi, \Phi)_{0}$, and suppose $(g, \eta, \xi, \Phi) \in \mathcal{S}\left(\xi, \bar{J}_{0}\right)$ has vanishing reduced scalar curvature $s_{g}^{G}=0$. Then there is a neighborhood of zero $\mathcal{V} \subset \mathfrak{B} \times \mathfrak{g}$ so that for $(t, \alpha) \in \mathcal{V}$ there is smooth Sasaki metric $g_{t, \alpha, \phi_{t, \alpha}}$ satisfying $s_{g_{t, \alpha, \phi_{t, \alpha}}}=0$. So that for each fixed $t \in \mathcal{B}$ close to zero, the space of extremal metrics is parametrized by a neighborhood of zero in $\mathfrak{g}$.

Proof. By the Lemma the relative Futaki invariant is nondegenerate. As above, define $K=\operatorname{ker} D_{g} \mathscr{S}$. Suppose $(0,0, \dot{\phi}) \in K$, then by (60) we have $\mathbb{L}_{g} \dot{\phi}=0$. So $\dot{\phi} \in \sqrt{-1} \mathcal{H}_{g}^{\mathfrak{z}^{\prime}}=\mathcal{H}_{g}^{\mathfrak{z}}$, and $\dot{\phi}=0$ since $\dot{\phi} \in W_{k+4,0}$. Therefore the projection $\varpi: K \rightarrow \mathfrak{g}, \varpi(t, \alpha, \phi)=\alpha$ is an isomorphism. We consider the map

$$
\mathscr{S} \times \varpi \circ \pi: \mathcal{V} \rightarrow \mathcal{B} \times W_{k, 0} \times \mathfrak{g},
$$

whose differential at zero is an isomorphism. The proof then follows from the inverse function theorem as in Theorem 32 .

\subsection{Sasaki-Einstein case.}

4.3.1. Necessary condition for a Sasaki-Einstein structure. Because of Corollary 29 we might as well assume $G=T^{r}$ and $T^{r} \subseteq G^{\prime}$ is a maximal torus. We recall the necessary condition for $(g, \eta, \xi, \Phi)$ to admit a transverse Kähler deformation to a Sasaki-Einstein structure, or rather a structure which is transversally KählerEinstein, $\operatorname{Ric}^{T}=\tau g^{T}, \tau>0$. The following necessary conditions are well known. See [16] or [21].

Proposition 35. The following conditions are equivalent.

(i) $\frac{\tau}{2 \pi}\left[\omega^{T}\right]=c_{1}^{b}\left(\mathscr{F}_{\xi}\right)$ in $H_{b}^{2}\left(M / \mathscr{F}_{\xi}\right)$ for $\tau>0$.

(ii) The class $c_{1}^{b}\left(\mathscr{F}_{\xi}\right)$ is represented by a positive $(1,1)$ basic form and $c_{1}(D)=0$.

(iii) There exists a nowhere vanishing holomorphic $(m+1,0)$-form $\Omega \in \Gamma\left(\Lambda^{1,1} C(M)\right)$ for which $\mathcal{L}_{\xi} \Omega=\sqrt{-1} \frac{\tau}{2} \Omega$. If $M$ is not simply connected, then we may have to take $\Omega$ to be multi-valued, or $\Omega \in \Gamma\left(\Lambda^{1,1} C(M)\right)^{\otimes \ell}$.

Remark 4.36. Note that the conditions imply $\pi_{1}(M)$ must be finite. The transverse Calabi-Yau theorem [13] implies the existence of a transverse Kähler deformation to transversal metric with $\operatorname{Ric}^{T}>0$. After a possible homothety, this lifts to a Sasaki structure with $\mathrm{Ric}_{g}>0$, and the claim follows from Myers' Theorem.

Proof. In order to prove the equivalence of (1i) and (iii) consider the Gysin sequence [3, Ch. 7]

$$
0 \rightarrow H_{b}^{0}\left(M / \mathscr{F}_{\xi}\right) \stackrel{\delta}{\longrightarrow} H_{b}^{2}\left(M / \mathscr{F}_{\xi}\right) \stackrel{\iota}{\longrightarrow} H^{2}(M, \mathbb{R}) \rightarrow \cdots,
$$

where $\delta \alpha=[\alpha d \eta]_{b}$. If we have (ii), then $\iota\left(c_{1}^{b}\left(\mathscr{F}_{\xi}\right)\right)=0$. But this represents $c_{2}(D)$. If (iii) holds, then again $\iota\left(c_{1}^{b}\left(\mathscr{F}_{\xi}\right)\right)=0$, so there exists an $\alpha \in \mathbb{R}$ with $\delta(\alpha)=2 \alpha\left[\omega^{T}\right]=$ $c_{1}^{b}\left(\mathscr{F}_{\xi}\right.$. But by assumption we must have $\alpha>0$. 
Supposing (iii) we have

$$
\left(\frac{\sqrt{-1}}{2}\right)^{m+1}(-1)^{m(m+1) / 2} \Omega \wedge \bar{\Omega}=\exp (h) \frac{1}{(m+1) !} \omega^{m+1},
$$

with $\omega$ the Kähler form of $(C(M), \bar{g})$ and $h \in C^{\infty}(C(M))$. Taking the Lie derivative $\mathcal{L}_{\xi}$ of (63), we see the condition in (iii) implies $\mathcal{L}_{\xi} h=0$. We make a homothetic deformation $\left(g_{a}, \eta_{a}, \xi_{a}, \Phi\right)$ of $(g, \eta, \xi, \Phi)$ with $a=\frac{\tau}{2 m+2}$, i.e. $\eta_{a}=a \eta, \xi_{a}=\frac{1}{a} \xi$ and $g_{a}=a g+\left(a^{2}-a\right) \eta \otimes \eta$. We use our original notation for this homothetic Sasaki structure, then we have $\mathcal{L}_{\xi} \Omega=\sqrt{-1}(m+1) \Omega$. Then applying $\mathcal{L}_{r \partial_{r}}$ to (63), with the new Sasaki structure, since $\mathcal{L}_{r \partial_{r}} \omega=2 \omega$, we have $\mathcal{L}_{r \partial_{r}} h=0$. Thus $h \in C_{b}^{\infty}(M)$ is basic, and the Ricci form $\rho$ of $(C(M), \bar{g})$ is

$$
\sqrt{-1} \partial \bar{\partial} h=\rho=\rho^{T}-(2 m+2) \omega^{T},
$$

which implies (ii) with $\tau=2 m+2$.

Conversely, assuming (ii) we make a homothetic transformation so that $\tau=$ $2 m+2$. Then the basic cohomology class $\left[\rho^{T}-(2 m+2) \omega^{T}\right]_{b}=0$, so the transverse $\partial \bar{\partial}$-Lemma [13] gives an $h \in C^{\infty}(M)_{b}$ satisfying (64). Define an Hermitian metric on $\Lambda^{1,1} C(M)$ by

$$
\|\Omega\|_{h}:=\left(\frac{\sqrt{-1}}{2}\right)^{m+1}(m+1) !(-1)^{m(m+1) / 2} \exp (-h) \frac{\Omega \wedge \bar{\Omega}}{\omega^{m+1}} .
$$

The curvature of the Chern connection of $\|\cdot\|_{h}$ is $\sqrt{-1} \partial \bar{\partial} h-\rho=0$. Therefore the universal cover $\varpi: \tilde{M} \rightarrow M$ has a parallel section $\Omega \in \Lambda^{1,1} C(\tilde{M})$.

Suppose Proposition 35 holds for $(g, \eta, \xi, \Phi)$ with $G \subseteq \operatorname{Aut}(g, \eta, \xi, \Phi)_{0}$. We assume $\tau=2 m+2$ for simplicity. Since $G$ is connected (ii) is preserved by $G$, and in (64) we may take $h \in C_{b}^{\infty}(M)$ to be $G$-invariant. Thus the metric $\|\cdot\|_{h}$ is $G$-invariant. Because $g^{*} \Omega$ is parallel and $\left\|g^{*} \Omega\right\|_{h}=1$ for $g \in G, g^{*} \Omega=\chi(g) \Omega$ with $\chi(g) \in \mathrm{U}(1)$. And

$$
\chi: G \rightarrow \mathrm{U}(1)
$$

is a character.

For the remainder of the section we suppose that $G=T \subset G^{\prime}=\operatorname{Aut}(g, \eta, \xi, \Phi)$ is a maximal torus. Then of course, $\mathfrak{z}=\mathfrak{z}^{\prime}=\mathfrak{g}$.

Definition 37. We define the characteristic hyperplane of a Sasaki structure $(g, \eta, \xi, \Phi)$ satisfying Proposition 35 to be the hyperplane $\mathcal{P}=\left\{X \in \mathfrak{g}: \chi_{*} X=\right.$ $\sqrt{-1}(m+1)\} \subset \mathfrak{g}$ containing $\xi$.

And define $\mathcal{Q}=\mathcal{P}-\xi=\left\{X \in \mathfrak{g}: \operatorname{ker} \chi_{*}\right\} \subset \mathfrak{g}$ to be the corresponding linear space.

For any $\xi_{\alpha}=\xi+\alpha \in \mathcal{P} \cap \mathfrak{g}^{+}$the Sasaki structure $\left(g_{\alpha}, \eta_{\alpha}, \xi_{\alpha}, \Phi_{\alpha}\right)$ with Reeb vector field $\xi_{\alpha}$ defined in (46) and (477) satisfies Proposition 35.

4.3.2. Volume functional and Futaki invariant. We will consider the space of Sasaki structures on $M$ considered in Section 3.2.1 depending on $\left(\xi_{\alpha}, \phi\right) \in \mathfrak{z}^{+} \times C^{\infty}(M)^{G}$ with Reeb vector field $\xi_{\alpha}=\xi+\alpha \in \mathfrak{z}^{+}$and $\eta_{\alpha, \phi}=\eta_{\xi_{\alpha}}+d^{c} \phi$. These Sasaki structures correspond to a space of Kähler cone metrics on $(C(M), I)$ with $G$ contained in the 
isometry group. We denote this space of Sasaki structures on $M$ by $\mathcal{S}(G, I)$. Just as in 21] we consider the volume functional

$$
\begin{aligned}
\mathcal{S}(G, I) & \stackrel{\text { Vol }}{\longrightarrow} \mathbb{R} \\
\left(g_{\alpha, \phi}, \eta_{\alpha, \phi}, \zeta_{\alpha}, \Phi_{\alpha, \phi}\right) & \mapsto \quad \int_{M} d \mu_{g_{\alpha, \phi}},
\end{aligned}
$$

where it can be shown that $\operatorname{Vol}\left(g_{\alpha, \phi}\right)=\frac{1}{m !} \int_{M} \eta_{\alpha, \phi} \wedge\left(\frac{1}{2} d \eta_{\alpha, \phi}\right)^{m}$ depends only on the Reeb vector field $\xi_{\alpha}$. See also [16. Thus (67) defines a functional

$$
\mathrm{Vol}: \mathfrak{g}^{+} \rightarrow \mathbb{R} \text {. }
$$

We will need the first and second variation formulae of Vol which were first calculated in 21. Let $\left\{\left(g(t), \eta_{t}, \xi_{t}, \Phi_{t}\right\}_{-\epsilon<t<\epsilon}\right.$ be a 1-parameter family of Sasaki structures in $\mathcal{S}(G, I)$ with $g(0)=g$ and $\dot{\xi}_{0}=X$, then

$$
D_{g} \operatorname{Vol}(X)=\left.\frac{d}{d t} \operatorname{Vol}(g(t))\right|_{t=0}=-(m+1) \int_{M} \eta(X) d \mu_{g} .
$$

For the second derivative, let $\left\{\left(g(t), \eta_{t}, \xi_{t}, \Phi_{t}\right\}_{-\epsilon<t<\epsilon}\right.$ be a 1-parameter family of Sasaki structures in $\mathcal{S}(G, I)$ with $g(0)=g$ and $\dot{\xi}_{0}=Y$, then

$$
\begin{aligned}
D_{g}^{2} \operatorname{Vol}(X, Y) & =-\left.(m+1) \frac{d}{d t}\left(\int_{M} \eta_{t}(X) d \mu_{g_{t}}\right)\right|_{t=0} \\
& =(m+1)(m+2) \int_{M} \eta(X) \eta(Y) d \mu_{g} .
\end{aligned}
$$

Therefore, Vol $: \mathfrak{g}^{+} \cap \mathcal{P} \rightarrow \mathbb{R}$ is strictly convex function on a convex polytope $\mathfrak{g}^{+} \cap \mathcal{P}$. Moreover, one can show that the integral in (67) goes to infinity as $\xi_{\alpha}$ approaches the boundary of $\mathfrak{C}_{\mathfrak{z}}=\mathfrak{g}^{+}$.

Proposition 38. Let $\left(g^{\prime}, \eta^{\prime}, \xi^{\prime}, \Phi^{\prime}\right) \in \mathcal{S}(G, I)$ have $\xi^{\prime} \in \mathcal{P}$. Then $\xi^{\prime}$ is a critical point for $\mathrm{Vol}: \mathfrak{g}^{+} \cap \mathcal{P} \rightarrow \mathbb{R}$ if and only if the Futaki invariant restricted to $\mathfrak{g}$ vanishes, $\left.\mathcal{F}_{\xi^{\prime}}\right|_{\mathfrak{g}} \equiv 0$.

Proof. We consider the following set of potentials for the transversely holomorphic vector fields $\pi(\mathfrak{g})^{1,0} \subseteq \mathfrak{h o l}^{T}\left(\xi^{\prime}, \bar{J}\right)_{0}$. Define

$$
\tilde{\mathcal{H}}_{g^{\prime}}:=\left\{\sqrt{-1} \eta^{\prime}(X) \mid X \in \mathcal{Q}\right\} \subset \mathcal{H}_{g^{\prime}}^{\mathfrak{g}} .
$$

We define the operator appearing in [15, with $h$ given in (64) and $\square_{b}=\frac{1}{2} \Delta_{b}$ the complex Laplacian,

$$
\left.\square_{b}^{h} u:=\square_{b} u-\partial^{\#} u\right\lrcorner \partial h .
$$

Note that $\square_{b}^{h}$ is self adjoint with respect to a weighted volume on $M$,

$$
\int_{M} \square_{b}^{h} u \bar{v} e^{h} d \mu_{g^{\prime}}=\int_{M} u \overline{\square_{b}^{h} v} e^{h} d \mu_{g^{\prime}}
$$

We say that a holomorphy potential $u_{X}$ is normalized if

$$
\int_{M} u_{X} e^{h} d \mu_{g^{\prime}}=0 .
$$

We will need the following result from [15] and [16].

Theorem 39. Suppose $\left(g^{\prime}, \eta^{\prime}, \xi^{\prime}, \Phi^{\prime}\right)$ satisfies Proposition 35 and $\square_{b}^{h}$ defined in (71). The eigenspace $\left\{u \in C_{b}^{\infty}(M, \mathbb{C}) \mid \square_{b}^{h} u=(2 m+2) u\right\}$ is isomorphic to the space of normalized holomorphy potentials. 
Let $X \in \mathcal{Q}$, and apply $\mathcal{L}_{I X}$ to (63) to get

$$
\begin{aligned}
0 & =(I X) h+\frac{1}{2} \Delta^{C(M)} r^{2} \eta^{\prime}(X) \\
& =(I X) h+\frac{1}{2}\left(\frac{1}{r^{2}} \Delta_{b}^{M}-\frac{\partial^{2}}{\partial r^{2}}-\frac{(2 m+1)}{r} \frac{\partial}{\partial r}\right) r^{2} \eta^{\prime}(X) \\
& =(I X) h+\frac{1}{2} \Delta_{b}^{M} \eta^{\prime}(X)-(2 m+2) \eta^{\prime}(X) \\
& =\square_{b}^{h} u-(2 m+2) u .
\end{aligned}
$$

And it follows that the space of normalized holomorphy potentials for $\pi(\mathfrak{g})^{1,0} \subseteq$ $\mathfrak{h o l}^{T}\left(\xi^{\prime}, \bar{J}\right)_{0}$ is $\tilde{\mathcal{H}}_{g^{\prime}}$.

We have $\frac{1}{2} \bar{J} \operatorname{grad} \eta^{\prime}(X)=X$ and

$$
\begin{aligned}
\mathcal{F}_{\xi^{\prime}}(X) & =\int_{M} d^{c} h(X) d \mu_{g^{\prime}} \\
& =\int_{M}-\bar{J} X h-\frac{1}{2} \Delta_{b} \eta^{\prime}(X) d \mu_{g^{\prime}} \\
& =-(2 m+2) \int_{M} \eta^{\prime}(X) d \mu_{g^{\prime}},
\end{aligned}
$$

from which the Proposition follows.

4.3.3. Deformations of Sasaki-Einstein structures. We now consider a $G$-equivariant deformation $\left(\mathscr{F}_{\xi}, \bar{J}_{t}\right)_{t \in \mathcal{B}}$. It is useful that the Kuranishi space of Section 3.1 .1 is always smooth because of the following.

Proposition 40. Suppose that the conditions of Proposition 35 hold, or more generally, $c_{1}^{b}>0$. Then $H_{\bar{\partial}_{b}}^{2}\left(\mathcal{A}^{0, \bullet}\right)=0$. Thus the Kuranishi space $\mathcal{V}$ and the submanifold of G-equivariant deformations $\mathcal{V}^{G}$ are smooth.

Proof. Using harmonic theory for the Laplacian $\Delta_{\bar{\partial}_{b}}=\bar{\partial}_{b} \bar{\partial}_{b}^{*}+\bar{\partial}_{b}^{*} \bar{\partial}_{b}$ associated with the complex (41) one can prove Serre duality using the same arguments as in [18] to get

$$
\begin{aligned}
H_{\bar{\partial}_{b}}^{2}\left(\mathcal{A}^{0, \bullet}\right) & \cong H_{\bar{\partial}_{b}}^{m-2}\left(\mathcal{A}^{m, \bullet} \otimes \Lambda_{b}^{1,0}\right) \\
& \cong H_{\bar{\partial}_{b}}^{m-2}\left(\mathcal{A}^{1, \bullet} \otimes \Lambda_{b}^{m, 0}\right)
\end{aligned}
$$

By our assumption $\Lambda_{b}^{m, 0}$ admits a connection with negative curvature. Again using harmonic representatives, the proof of Kodaira-Nakano vanishing in [18 works in this situation and we get the last term in (75) is zero because $(m-2)+1<m$.

Recall that by Proposition 21 the existence of Sasaki structures on a deformation in this case is unobstructed. We have a family $\left(g_{t}, \eta_{t}, \xi, \Phi_{t}\right) \in \mathcal{S}\left(\xi, \bar{J}_{t}\right), t \in \mathcal{B}$ of compatible Sasaki structures with $\left(g_{0}, \eta_{0}, \xi_{0}, \Phi_{0}\right)$ satisfying Proposition 35, with $\tau=2 m+2$. Since $c_{1}^{b}\left(\mathscr{F}_{\xi}, \bar{J}_{t}\right)$ is unchanged under deformation of $\bar{J}_{t}$, condition (il) of the Proposition holds for all $t \in \mathcal{B}$. We can define $h_{t} \in C_{b}^{\infty}(M)$ depending smoothly on $t \in \mathcal{B}$ by

$$
h_{t}=2 G_{g_{t}^{T}}\left(\omega_{t}^{T}, \rho_{t}^{T}-(2 m+2) \omega_{t}^{T}\right)_{g_{t}^{T}}=2 G_{g_{t}^{T}}\left(s_{t}^{T}-s_{0}^{T}\right),
$$

where $G_{g_{t}^{T}}$ is the Green's function of $g_{t}^{T}$. By taking parallel displacement from a fixed point with respect to the flat Chern connection of $\|\cdot\|_{h_{t}}$ on $\Lambda^{m+1,0}(C(M))$, we get a smooth family of holomorphic $(m+1,0)$-forms $\Omega_{t}$ on the family of cones 
$C\left(M_{t}\right)=\left(C(M), I_{t}\right)$. Then for each $t \in \mathcal{B}$ as in (66) we have a character $\chi_{t}: G \rightarrow$ $\mathrm{U}(1)$. Since the characters on $G$ is discrete lattice, $\chi_{t}$ is independent of $t \in \mathcal{B}$. It follows that the characteristic hyperplane $\mathcal{P} \subset \mathfrak{g}$ is independent of $t \in \mathcal{B}$.

Corollary 41. Let $(g, \eta, \xi, \Phi)$ be a Sasaki-Einstein structure, and suppose that $\left(\mathscr{F}_{\xi}, \bar{J}_{t}\right)_{t \in \mathcal{B}}$ is a $G$-equivariant deformation, where $G \subseteq G^{\prime}=\operatorname{Aut}(g, \eta, \xi, \Phi)_{0}$ is a maximal torus. Then there is a neighborhood $U \subset \mathcal{B}$ so that for $t \in U$ there is a unique $\alpha_{t} \in \mathcal{Q} \subset \mathfrak{g}$ and a $\phi_{t} \in C^{\infty}(M)^{G}$ so that $g_{t, \alpha_{t}, \phi_{t}}$ is Sasaki-Einstein.

Proof. We modify the map (59) Let $\tilde{\mathcal{V}}=\mathcal{U} \cap \mathcal{B} \times \mathcal{Q} \times W_{k+4,0}$. Then we define a map

$$
\begin{array}{cccc}
\tilde{\mathscr{S}}: & \tilde{\mathcal{V}} & \rightarrow & \mathcal{B} \times Q^{*} \times W_{k, 0} \\
(t, \alpha, \phi) & \mapsto & \left(t, \mathcal{F}(\alpha), \pi_{0}^{W}\left(s_{t, \alpha, \phi}^{G}\right)\right),
\end{array}
$$

where $\mathcal{F}(\alpha) \in Q^{*}$ is defined by $\mathcal{F}(\alpha)(X):=-\int_{M} \eta_{\alpha, \phi}(X) d \mu_{g_{\alpha, \phi}}$. Then $D_{g} \tilde{\mathscr{S}}$ is given by Lemma 30 with the exception of $D_{g} \mathcal{F}(\dot{\alpha})$ which is given by (70)

$$
D_{g} \mathcal{F}(\dot{\alpha})(X)=(m+2) \int_{M} \eta(\dot{\alpha}) \eta(X) d \mu_{g}, \quad X \in \mathcal{Q} .
$$

It is routine to check that

$$
D_{g} \tilde{\mathscr{S}}: \mathcal{B} \times \mathcal{Q} \times W_{k+4,0} \rightarrow \mathcal{B} \times Q^{*} \times W_{k, 0}
$$

is an isomorphism. By the inverse function theorem there is a neighborhood $\mathcal{U} \subset$ $\mathcal{B} \times Q^{*} \times W_{k, 0}$ on which $\tilde{\mathscr{S}}^{-1}$ is defined. Then with $U=\mathcal{U} \cap \mathcal{B} \times\{0\} \times\{0\}$ we set $\left(t, \alpha_{t}, \phi_{t}\right)=\tilde{\mathscr{S}}^{-1}(t, 0,0)$ for $t \in U$. And $g_{t, \alpha_{t}, \phi_{t}}$ is a Sasaki-extremal metric. Since $s_{g_{t, \alpha_{t}, \phi_{t}}}^{G}=0$ for $t \in U$ we have $\bar{J}_{t} \operatorname{grad} s_{g_{t, \alpha_{t}, \phi_{t}}} \in \pi(\mathfrak{g}) \subset \mathfrak{h o l}^{T}\left(\xi+\alpha_{t}, \bar{J}_{t}\right)_{0}$.

We denote the metric $g_{t, \alpha_{t}, \phi_{t}}$ by $g_{t}$ for brevity. Let $h_{t} \in C_{b}^{\infty}(M)^{G}$ satisfy (64), then $\Delta_{b} h_{t}=s_{g_{t}}^{T}-s_{0}^{T}=s_{g_{t}}-s_{0}$, where $s_{0}^{T}=(2 m+2)(2 m)$ and $s_{0}$ are the averages of $s_{g_{t}}^{T}$ and $s_{g_{t}}$. By Proposition 38 the Futaki invariant $\left.\mathcal{F}_{\xi+\alpha_{t}}\right|_{\mathfrak{g}} \equiv 0$, and with $X=\bar{J} \operatorname{grad} s_{g_{t}}$ we have

$$
\begin{aligned}
0=\mathcal{F}_{\xi+\alpha_{t}}(X) & =\int_{M} d^{c} h_{t}(X) d \mu_{g_{t}} \\
& =\int_{M}\left(d s_{g_{t}}, d h_{t}\right) d \mu_{g_{t}} \\
& =\int_{M}\left(s_{g_{t}}, \Delta_{b} h_{t}\right) d \mu_{g_{t}} \\
& =\int_{M}\left(s_{g_{t}}, s_{g_{t}}-s_{0}\right) d \mu_{g_{t}} \\
& =\int_{M}\left\|s_{g_{t}}-s_{0}\right\|^{2} d \mu_{g_{t}} .
\end{aligned}
$$

So $s_{g_{t}}-s_{0}=0$ and $h_{t}$ is constant, therefore $g_{t, \alpha_{t}, \phi_{t}}$ is Sasaki-Einstein.

\section{EXAmples}

We describe a family of examples of 7-manifolds on which we can apply Corollary 34 and Corollary 41 to give new families of Sasaki-extremal and Sasaki-Einstein metrics. More details will appear in [29]. These examples are deformations of 3Sasaki manifolds that first appeared in the work of C. Boyer, K. Galicki, B. Mann, and E. Reese [4. 
Definition 42. A Riemannian manifold $(M, g)$ is 3-Sasaki if the metric cone $(C(M), \bar{g})$ is hyperkähler, i.e. $\bar{g}$ admits compatible almost complex structures $J_{\alpha}, \alpha=$ $1,2,3$ such that $\left(C(M), \bar{g}, J_{1}, J_{2}, J_{3}\right)$ is a hyperkähler structure. Equivalently, $\operatorname{Hol}(C(M)) \subseteq \mathrm{Sp}(m)$.

A consequence of the definition is that $(M, g)$ is equipped with three Sasaki structures $\left(\xi_{i}, \eta_{i}, \phi_{i}\right), i=1,2,3$. The Reeb vector fields $\xi_{k}, k=1,2,3$ are orthogonal and satisfy $\left[\xi_{i}, \xi_{j}\right]=2 \varepsilon^{i j k} \xi_{k}$, where $\varepsilon^{i j k}$ is anti-symmetric in the indices $i, j, k \in\{1,2,3\}$ and $\varepsilon^{123}=1$.

The Reeb vector fields $\xi_{k}, k=1,2,3$ generate an action of $\mathrm{Sp}(1)$ or $\mathrm{SO}(3)$. A 3 -Sasaki manifold $M$ comes with a family of related geometries. The maps are labeled with their generic fibers.

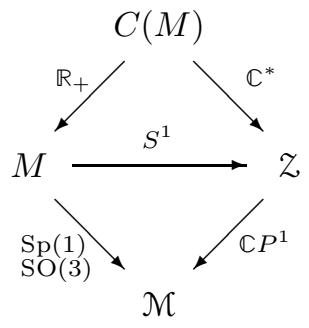

The leaf space $\mathcal{Z}$ is an orbifold with complex contact structure, while $\mathcal{M}$ is a quaternionic-Kähler orbifold. This intimate relation with other more well known geometries is probably the reason 3-Sasaki manifolds have not been studied as much quaternionic-Kähler manifolds. For more details see [2]

A 3-Sasaki manifolds $(M, g), \operatorname{dim} M=4 m-1$, is toric if there is a $T^{m} \subseteq$ $\operatorname{Aut}\left(M, g, \xi_{1}, \xi_{2}, \xi_{3}\right)$. Toric 3-Sasaki manifolds have been constructed from 3-Sasaki quotients by torus actions on $S^{4 n-1}$, with the 3-Sasaki structure given by right multiplication by $\operatorname{Sp}(1)$. A subtorus $T^{k} \subset T^{n}$ is determined by a weight matrix $\Omega_{k, n} \in \operatorname{Mat}(k, n, \mathbb{Z})$. There are conditions on $\Omega[4$ that imply the moment map $\mu: S^{4 n-1} \rightarrow\left(\mathfrak{t}^{k}\right)^{*} \otimes \mathbb{R}^{3}$ is a submersion, and further that the quotient

$$
M_{\Omega_{k, n}}=S^{4 n-1} / / T^{k}=\mu^{-1}(0) / T^{k}
$$

is smooth.

When $n=k+2$ it was shown in [4 that there are infinitely many weight matrices in $\operatorname{Mat}(k, n, \mathbb{Z})$ for $k \geq 1$ giving infinitely many 7 -manifolds $M_{\Omega_{k, n}}$ for each $b_{2}\left(M_{\Omega_{k, n}}\right)=k \geq 1$.

If $b_{2}(M) \geq 1$, then the maximal torus of Sasaki automorphisms, $T^{3} \subset \operatorname{Aut}\left(M, \xi_{1}\right)$, is 3 -dimensional. And if $b_{2}(M) \geq 2$, then the connected component of the identity of $\operatorname{Isom}(g)=T^{2} \times \mathrm{Sp}(1)$ or $T^{2} \times \mathrm{SO}(3)$, where the second factor is generated by $\left\{\xi_{1}, \xi_{2}, \xi_{3}\right\}$.

Proposition 43 (29]). Let $(M, g)$ be a toric 3-Sasaki 7-manifold. Then after fixing one of the Sasaki structures $\left(g, \eta_{1}, \xi_{1}, \Phi_{1}\right)$ with foliation $\mathscr{F}_{\xi_{1}}$ we have

$$
H_{\bar{\partial}_{b}}^{1}\left(\mathcal{A}^{0, \bullet}\right)=H_{\bar{\partial}_{b}}^{1}\left(\mathcal{A}^{0, \bullet}\right)^{T^{3}}=b_{2}(M)-1=k-1 .
$$

By Proposition 40 there is a smooth Kuranishi space $\mathcal{B}$ of deformations of $\left(\mathscr{F}_{\xi_{1}}, \bar{J}\right)$ equivariant with respect to $T^{3}$. One can further show that the family 


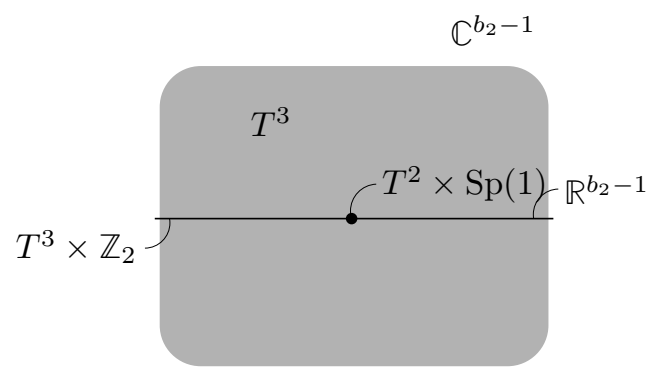

Figure 1. Space of Sasaki-Einstein metrics

$\mathcal{B}$ is effective [29]. Let $\mathfrak{t}$ denote the Lie algebra of $T^{3}$. By Corollary 34 there is a neighborhood $N \subset \mathcal{B} \times \mathfrak{t}$ parametrizing a space of dimension $b_{2}(M)+2$ of Sasaki-extremal metrics. And by Corollary 41 there is a $b_{2}(M)-1$-dimensional submanifold $U \subset N$ parametrizing a space of Sasaki-Einstein metrics. Note that all these Sasaki-extremal structures satisfy Proposition 35, while only the submanifold $U \subset N$ of Einstein metrics and their homotheties have constant scalar curvature since the rest have non-vanishing Futaki invariant. See Figure 1 which shows the isometry groups. Note that the origin is 3-Sasaki while the other metrics are just Sasaki-Einstein. It is well known that 3-Sasaki structures are non-deformable, but these are the first examples known to the author of 3-Sasaki structures contained in families of Sasaki-Einstein structures.

Recall that a 3-Sasaki manifold $M$ with $\operatorname{dim} M=4 m-1$ admits $m+1$ Killing spinors whereas a simply connected Sasaki-Einstein, non-3-Sasaki, metric admits 2. So these families give examples of Einstein metrics admitting 3 Killing spinors with deformations to Einstein metrics admitting only 2. These properties are explored further in [29].

\section{REFERENCES}

[1] Arthur L. Besse. Einstein manifolds, volume 10 of Ergebnisse der Mathematik und ihrer Grenzgebiete (3) [Results in Mathematics and Related Areas (3)]. Springer-Verlag, Berlin, 1987.

[2] Charles Boyer and Krzysztof Galicki. 3-Sasakian manifolds. In Surveys in differential geometry: essays on Einstein manifolds, Surv. Differ. Geom., VI, pages 123-184. Int. Press, Boston, MA, 1999.

[3] Charles P. Boyer and Krzysztof Galicki. Sasakian geometry. Oxford Mathematical Monographs. Oxford University Press, Oxford, 2008.

[4] Charles P. Boyer, Krzysztof Galicki, Benjamin M. Mann, and Elmer G. Rees. Compact 3Sasakian 7-manifolds with arbitrary second Betti number. Invent. Math., 131(2):321-344, 1998.

[5] Charles P. Boyer, Krzysztof Galicki, and Santiago R. Simanca. Canonical Sasakian metrics. Comm. Math. Phys., 279(3):705-733, 2008.

[6] Charles P. Boyer, Krzysztof Galicki, and Santiago R. Simanca. The Sasaki cone and extremal Sasakian metrics. In Riemannian topology and geometric structures on manifolds, volume 271 of Progr. Math., pages 263-290. Birkhäuser Boston, Boston, MA, 2009.

[7] Eugenio Calabi. Extremal Kähler metrics. In Seminar on Differential Geometry, volume 102 of Ann. of Math. Stud., pages 259-290. Princeton Univ. Press, Princeton, N.J., 1982. 
[8] Eugenio Calabi. Extremal Kähler metrics. II. In Differential geometry and complex analysis, pages 95-114. Springer, Berlin, 1985.

[9] X. X. Chen and G. Tian. Geometry of Kähler metrics and foliations by holomorphic discs. Publ. Math. Inst. Hautes Études Sci., (107):1-107, 2008.

[10] Suzana Falcão B. de Moraes and Carlos Tomei. Moment maps on symplectic cones. Pacific J. Math., 181(2):357-375, 1997.

[11] A. El Kacimi Alaoui and B. Gmira. Stabilité du caractère kählérien transverse. Israel J. Math., 101:323-347, 1997.

[12] A. El Kacimi-Alaoui and M. Nicolau. Déformations des feuilletages transversalement holomorphes à type différentiable fixe. Publ. Mat., 33(3):485-500, 1989.

[13] Aziz El Kacimi-Alaoui. Opérateurs transversalement elliptiques sur un feuilletage riemannien et applications. Compositio Math., 73(1):57-106, 1990.

[14] A. Futaki. An obstruction to the existence of Einstein Kähler metrics. Invent. Math., 73(3):437-443, 1983.

[15] Akito Futaki. Kähler-Einstein metrics and integral invariants, volume 1314 of Lecture Notes in Mathematics. Springer-Verlag, Berlin, 1988.

[16] Akito Futaki, Hajime Ono, and Guofang Wang. Transverse Kähler geometry of Sasaki manifolds and toric Sasaki-Einstein manifolds. J. Differential Geom., 83(3):585-635, 2009.

[17] J. Girbau. A versality theorem for transversely holomorphic foliations of fixed differentiable type. Illinois J. Math., 36(3):428-446, 1992.

[18] Phillip Griffiths and Joseph Harris. Principles of algebraic geometry. Wiley-Interscience [John Wiley \& Sons], New York, 1978. Pure and Applied Mathematics.

[19] C. LeBrun and S. R. Simanca. Extremal Kähler metrics and complex deformation theory. Geom. Funct. Anal., 4(3):298-336, 1994.

[20] Claude LeBrun and Santiago R. Simanca. On the Kähler classes of extremal metrics. In Geometry and global analysis (Sendai, 1993), pages 255-271. Tohoku Univ., Sendai, 1993.

[21] Dario Martelli, James Sparks, and Shing-Tung Yau. Sasaki-Einstein manifolds and volume minimisation. Comm. Math. Phys., 280(3):611-673, 2008.

[22] Hiraku Nozawa. Deformation of sasakian metrics. arXiv:0809.4699v5, Oct 2011.

[23] Barrett O'Neill. The fundamental equations of a submersion. Michigan Math. J., 13:459-469, 1966.

[24] Barrett O'Neill. Semi-Riemannian geometry, volume 103 of Pure and Applied Mathematics. Academic Press Inc. [Harcourt Brace Jovanovich Publishers], New York, 1983. With applications to relativity.

[25] Yann Rollin, Santiago R. Simanca, and Carl Tipler. Stability of extremal metrics under complex deformations. arXiv:1107.0456 4, July 2011.

[26] R. Schoen. On the conformal and CR automorphism groups. Geom. Funct. Anal., 5(2):464481, 1995.

[27] Santiago R. Simanca. A K-energy characterization of extremal Kähler metrics. Proc. Amer. Math. Soc., 128(5):1531-1535, 2000.

[28] Santiago R. Simanca. Heat flows for extremal Kähler metrics. Ann. Sc. Norm. Super. Pisa Cl. Sci. (5), 4(2):187-217, 2005.

[29] Craig van Coevering. Deformations of the Killing spinor equation on Sasaki-Einstein and 3-Sasaki manifolds. to appear, 2012.

Max-Planck-Institut für Mathematik, Vivatsgasse 7, 53111 Bonn Germany

E-mail address: craigvan@mpim-bonn.mpg.de 